Acta Crystallographica Section C

Crystal Structure

Communications

ISSN 0108-2701

Editor: George Ferguson

\title{
Potassium 3-Dithiocarboxy-3-aza-5-aminopentanoate Dihydrate
}

B. Prelesnik, K. Andjelković, Z. Markovic, T. Sabo and S. Trifunovic

This electronic document was scanned from an archival copy of material deposited to accompany a paper published in an IUCr journal. In many cases the only accessible copy was a microfilm of a poor-quality original. 
values of $10 *$ Fobs and $10 *$ Fold

KA 1221

Page 1

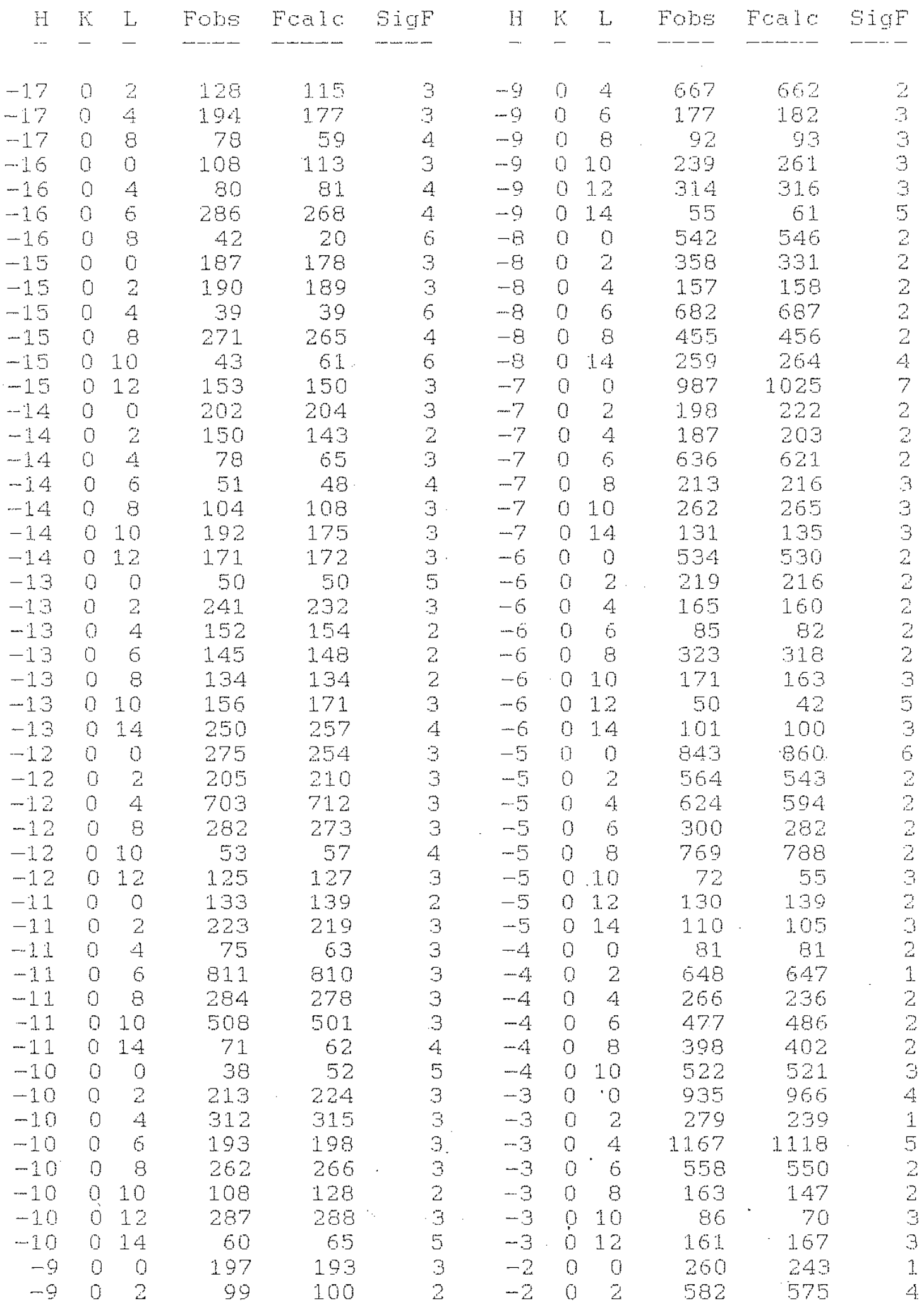
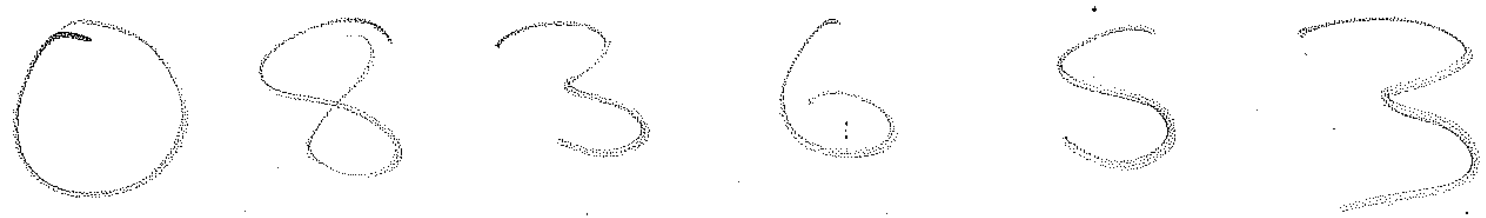
Values of 10*Fobs and 10*Foalo

Page 2

\begin{tabular}{|c|c|c|c|c|c|c|c|c|c|c|}
\hline $\mathrm{H}$ & $\begin{array}{ll}\mathrm{K} & \mathrm{L} \\
- & -\end{array}$ & Fobs & Fcalc & $S i g F$ & H & $K$ & L & Fobs & Foalc & $\operatorname{SigF}$ \\
\hline-2 & 4 & 422 & 423 & 2 & 5 & 0 & 2 & 137 & 153 & 2 \\
\hline-2 & 6 & 284 & 300 & 2 & 5 & 0 & 4 & 238 & 237 & 2 \\
\hline-2 & 8 & 479 & 487 & 2 & 5 & 0 & 6 & 318 & 309 & 3 \\
\hline-2 & 010 & 196 & 207 & 3 & 5 & 0 & 9 & 159 & 161 & 3 \\
\hline-2 & 012 & 136 & 133 & 2 & 5 & 0 & 10 & 82 & 77 & 3 \\
\hline-2 & 014 & 95 & 106 & 3 & 5 & 0 & 12 & 67 & 73 & 5 \\
\hline-1 & 0 & 203 & 219 & 1. & 6 & 0 & 0 & 532 & 530 & 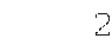 \\
\hline-1 & 2 & 362 & 333 & 1 & 6 & 0 & 2 & 725 & 737 & 2 \\
\hline-1 & 4 & 399 & 406 & 2 & 6 & 0 & 3 & 62 & 0 & 3 \\
\hline-1 & 6 & 244 & 251 & 2 & 5 & 0 & 6 & 173 & 181 & 3 \\
\hline-1 & 8 & 37 & 53 & 5 & 6 & 0 & 8 & 60 & 46 & 4 \\
\hline-1 & 010 & 123 & 106 & 2 & 6 & 0 & 10 & 55 & 61 & 5 \\
\hline-1 & 012 & 39 & 41 & 6 & 7 & 0 & 0 & 993 & 1025 & 7 \\
\hline-1 & $0 \quad 14$ & 181 & 182 & 3 & 7 & 0 & 4 & 181 & 179 & 3 \\
\hline 0 & 2 & 533 & 538 & 4 & 7 & 0 & 6 & 58 & 61 & 4 \\
\hline 0 & 4 & 202 & 215 & 2 & 7 & 0 & 8 & 46 & 52 & 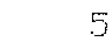 \\
\hline 0 & 6 & 110 & 118 & 2 & 7 & 0 & 10 & 98 & 90 & 3 \\
\hline 0 & 8 & 369 & 368 & 2 & 8 & 0 & 0 & 542 & 546 & 2 \\
\hline 0 & $0 \quad 10$ & 233 & 237 & 3 & 8 & 0 & 2 & 294 & 308 & 3 \\
\hline 0 & 012 & 47 & 36 & 5 & 8 & 0 & 4 & 121 & 127 & 2 \\
\hline 0 & 014 & 63 & 63 & 5 & 8 & 0 & 6 & 120 & 114 & 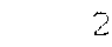 \\
\hline 1 & 0 & 199 & 219 & 1 & 8 & 0 & 10 & 216 & 209 & 3 \\
\hline 1 & 2 & 1326 & 1315 & 4 & 9 & 0 & 0 & 191 & 193 & 3 \\
\hline 1 & 4 & 231 & 256 & 2 & 9 & 0 & 2 & 204 & 214 & 3 \\
\hline 1 & 6 & 553 & 538 & 2 & 9 & 0 & 4 & 150 & 138 & 2 \\
\hline 1 & 8 & 99 & 95 & 2 & 10 & 0 & 0 & 46 & 52 & 4 \\
\hline 1 & 010 & 512 & 541 & 3 & 10 & 0 & 2 & 69 & 62 & 3 \\
\hline 1 & 012 & 134 & 142 & 2 & 10 & 0 & 4 & 79 & 80 & 3 \\
\hline 1. & 014 & 46 & 43 & 6 & 10 & 0 & 6 & 75 & 67 & 4 \\
\hline 2 & 0 & 261 & 243 & 1 & 10 & 0 & 8 & 125 & 118 & 3 \\
\hline 2 & 2 & 993 & 996 & 5 & 11 & 0 & 0 & 234 & 139 & 2 \\
\hline 2 & 4 & 300 & 289 & 2 & 11 & 0 & 2 & 183 & 171 & 3 \\
\hline 2 & 6 & 44 & 63 & 4 & 11 & 0 & 4 & 135 & 132 & . \\
\hline 2 & 010 & 65 & 65 & 4 & 11 & 0 & 6 & 58 & 60 & 5 \\
\hline 2 & 012 & 205 & 217 & 3 & 12 & 0 & 0 & 272 & 254 & 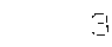 \\
\hline 3 & 0 & 931 & 966 & 4 & 12 & 0 & 2 & 234 & 226 & 3 \\
\hline 3 & 2 & 503 & 519 & 2 & 12 & 0 & 4 & 280 & 266 & 3 \\
\hline 3 & 0 & 201 & 223 & 2 & 12 & 0 & 6 & 161 & 145 & 2 \\
\hline 3 & 0 & 426 & 410 & 2 & 13 & 0 & a & 46 & 50 & 5 \\
\hline 3 & 8 & 382 & 397 & 3 & 13 & 0 & 2 & 97 & 87 & 3 \\
\hline 3 & 010 & 77 & 59 & 3 & 14 & 0 & 0 & 202 & 204 & 3 \\
\hline 3 & 012 & 181 & 183 & 3 & 14 & 0 & 2 & 70 & 55 & 4 \\
\hline 4 & 0 & 84 & 81 & 1 & 14 & 0 & 4 & 82 & 76 & $\Delta$ \\
\hline 4 & 2 & 493 & 522 & 2 & 15 & 0 & 0 & 186 & 178 & 3 \\
\hline 4 & 4 & 750 & 769 & 2 & 15 & 0 & 2 & 155 & 146 & 3 \\
\hline 4 & 6 & 237 & 246 & 3 & 16 & 0 & 0 & 1.14 & 113 & 3 \\
\hline 4 & 0 & 224 & 230 & 3 & -17 & 1. & 3 & 61 & 56 & 5 \\
\hline 4 & 010 & 232 & 231 & 3 & -17 & 1 & 4 & 119 & 107 & 3 \\
\hline 4 & 012 & 141 & 136 & 3 & -17 & 1. & 5 & 111 & 117 & \\
\hline 5 & 0 & 831 & 860 & 6 & -17 & 1 & 8 & 42 & 45 & 7 \\
\hline
\end{tabular}


Values of 10*Fobs and 10*Feald

Page 3

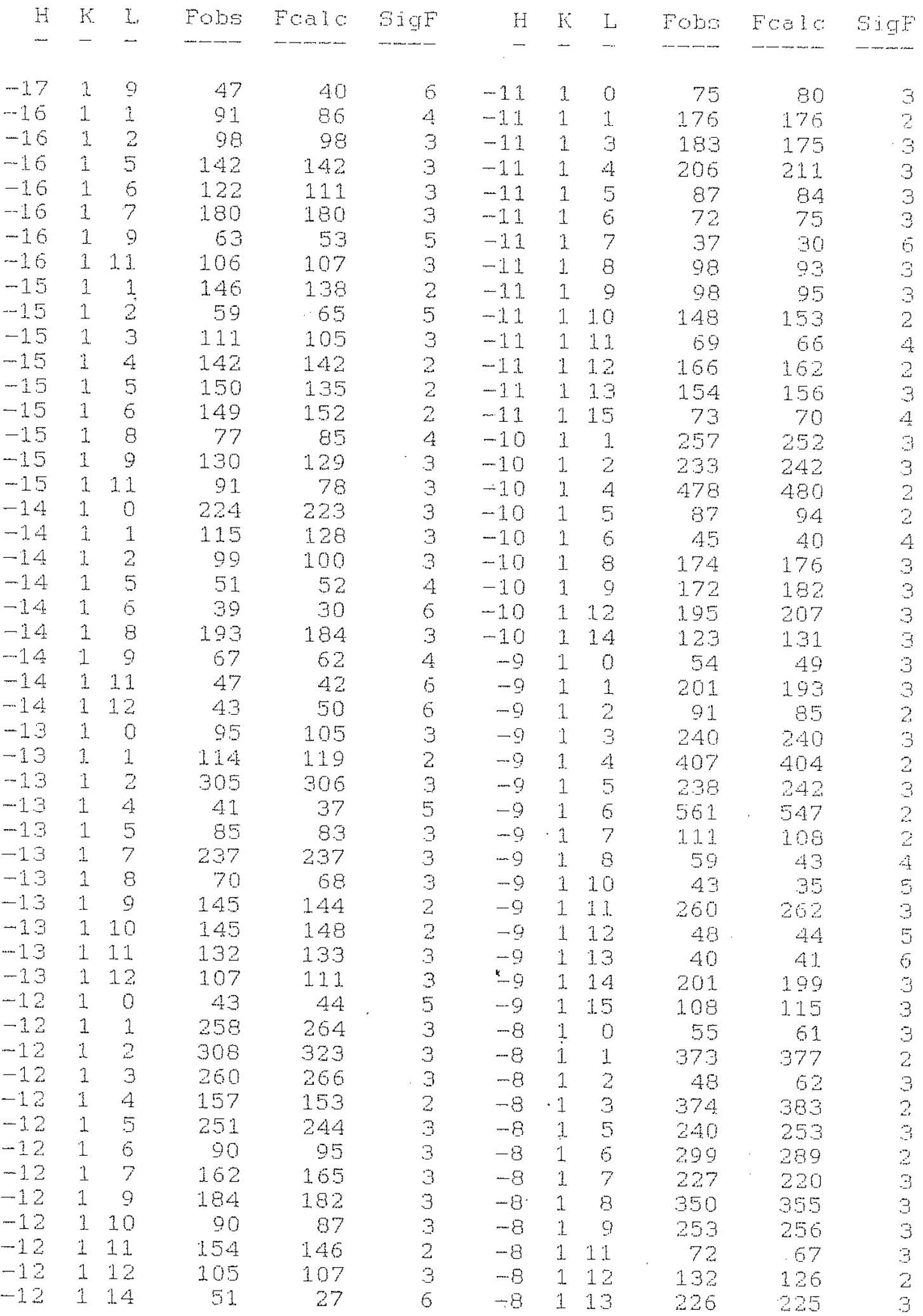


Values of $10 *$ Fobs and $10 *$ Fale

Pace 4

\begin{tabular}{|c|c|c|c|c|c|c|c|c|c|}
\hline $\mathrm{H}$ & 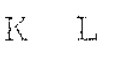 & Fobs & Foalo & $\operatorname{SigF}$ & $\mathrm{H}$ & L & Fobs & Fcalc & GigF \\
\hline- & - & - - & $-\cdots--$ & $\ldots-\ldots$ & - & - & $-\cdots$ & $--\cdots$ & - - \\
\hline-8 & 114 & 43 & 52 & 6 & -4 & 112 & 255 & 259 & 3 \\
\hline-7 & 10 & 379 & 413 & 2 & -4 & 113 & 203 & 208 & 3 \\
\hline-7 & 1 & 58 & 59 & 3 & -4 & 115 & 105 & 104 & 3 \\
\hline-7 & 2 & 294 & 264 & 2 & -3 & 10 & 189 & 182 & 2 \\
\hline-7 & 3 & 301 & 306 & 2 & -3 & 1 & 261 & 259 & 1 \\
\hline-7 & 4 & 326 & 319 & 2 & -3 & 2 & 58 & 34 & 2 \\
\hline-7 & 5 & 574 & 563 & 2 & -3 & 3 & 406 & 405 & 2 \\
\hline-7 & 6 & 268 & 260 & 2 & -3 & 4 & 375 & 349 & 2 \\
\hline-7 & 7 & 285 & 297 & 2 & -3 & .5 & 425 & 417 & 2 \\
\hline-7 & 8 & 470 & 480 & 2 & -3 & 7 & 378 & 375 & 2 \\
\hline-7 & 9 & 47 & 39 & 4 & -3 & 1 & 56 & 54 & 3 \\
\hline-7 & 110 & 52 & 48 & 4 & -3 & 1 & 122 & 124 & 2 \\
\hline-7 & 111 & 173 & 180 & 3 & -3 & 110 & 174 & 169 & 3 \\
\hline-7 & 12.2 & 132 & 143 & 2 & -3 & 112 & 67 & 78 & 4 \\
\hline-7 & 114 & 51 & 49 & 5 & -3 & 113 & 188 & 192 & 3 \\
\hline-7 & 115 & 135 & 134 & 3 & -3 & 114 & 87 & 99 & 3 \\
\hline-6 & 1 & 241 & 248 & 2 & -3 & 115 & 65 & 59 & 5 \\
\hline-6 & 2 & 532 & 549 & 2 & -3 & 10 & 468 & 478 & 1 \\
\hline-6 & 3 & 93 & 85 & 2 & -2 & 1 & 288 & 307 & 1 \\
\hline-6 & 4 & 54 & 29 & 3 & -2 & 2 & 375 & 351 & 1 \\
\hline-6 & 5 & 500 & 486 & 2 & -2 & 3 & 310 & 297 & 2 \\
\hline-6 & 6 & 238 & 240 & 2 & -2 & 1 & 919 & 894 & 5 \\
\hline-6 & 7 & 127 & 116 & 2 & -2 & 5 & 417 & 392 & 2 \\
\hline-6 & 9 & 237 & 233 & 3 & -2 & 6 & 447 & 452 & 2 \\
\hline-6 & 110 & 393 & 396 & 3 & -2 & 7 & 129 & 1.35 & 2 \\
\hline-6 & 111 & 266 & 270 & 3 & -2 & 8 & 362 & 370 & 2 \\
\hline-6 & 112 & 189 & 201 & 3 & -2 & 1 & 150 & 143 & 2 \\
\hline-6 & 11.4 & 150 & 148 & 2 & -2 & 110 & 190 & 202 & 3 \\
\hline-6 & 115 & 46 & 33 & 6 & -2 & 111 & 65 & 70 & 4 \\
\hline-5 & 1 & 787 & 811 & 6 & -2 & 112 & 135 & 14.5 & 2 \\
\hline-5 & 2 & 260 & 242 & 2 & -2 & 113 & 65 & 62 & 4 \\
\hline-5 & 3 & 336 & 327 & 2 & -2 & 21.4 & 88 & 81 & 3 \\
\hline-5 & 4 & 474 & 470 & 2 & -1 & 1 & 189 & 225 & 1 \\
\hline-5 & 5 & 80 & 102 & 2 & -1 & 1 & 294 & 283 & 1 \\
\hline-5 & 1 & 265 & 253 & 2 & -1 & 2 & 213 & 209 & 1 \\
\hline-5 & 8 & 261 & 280 & 3 & -1 & 1 & 359 & 347 & 1 \\
\hline-5 & 9 & 453 & 463 & 2 & -1 & 1 & 709 & 693 & 2 \\
\hline-5 & 110 & 209 & 215 & 3 & -1 & 1 & 535 & 518 & 2 \\
\hline-5 & 112 & 210 & 225 & 3 & -1 & 1 & 199 & 180 & 2 \\
\hline-5 & 113 & 104 & 109 & 3 & -1 & 1 & 306 & 283 & 2 \\
\hline-4 & 11 & 848 & 870 & 5 & -1 & 1.8 & 335 & 338 & 2 \\
\hline-4 & 2 & 612 & 590 & 1 & -1 & 19 & 82 & 89 & 3 \\
\hline-4 & 3 & 852 & 858 & 6 & -1 & 110 & 214 & 214 & 3 \\
\hline-4 & 4 & 314 & 282 & 2 & -1 & 1. 11 & 118 & $117^{\circ}$ & 2 \\
\hline-4 & 1 & 302 & 295 & 2 & -1 & 112 & 171 & 161 & 3 \\
\hline-4 & 6 & 339 & 313 & 2 & -1 & 113 & 157 & 161. & 2 \\
\hline-4 & 7 & 41 & 17 & 4 & -1 & 114 & 121 & 113 & 3 \\
\hline-4 & 9 & 170 & 154 & 3 & 0 & 1 & 148 & 151 & 1 \\
\hline-4 & 19 & 121 & 114 & 2 & 0 & 2 & 380 & 355 & 1 \\
\hline-4 & $1 \quad 11$ & 500 & 512 & 3 & 0 & 3 & 214 & 191 & 2 \\
\hline
\end{tabular}


Values of $10 \times$ Fobs and $10 \times \mathrm{Fcalc}$

Page 5

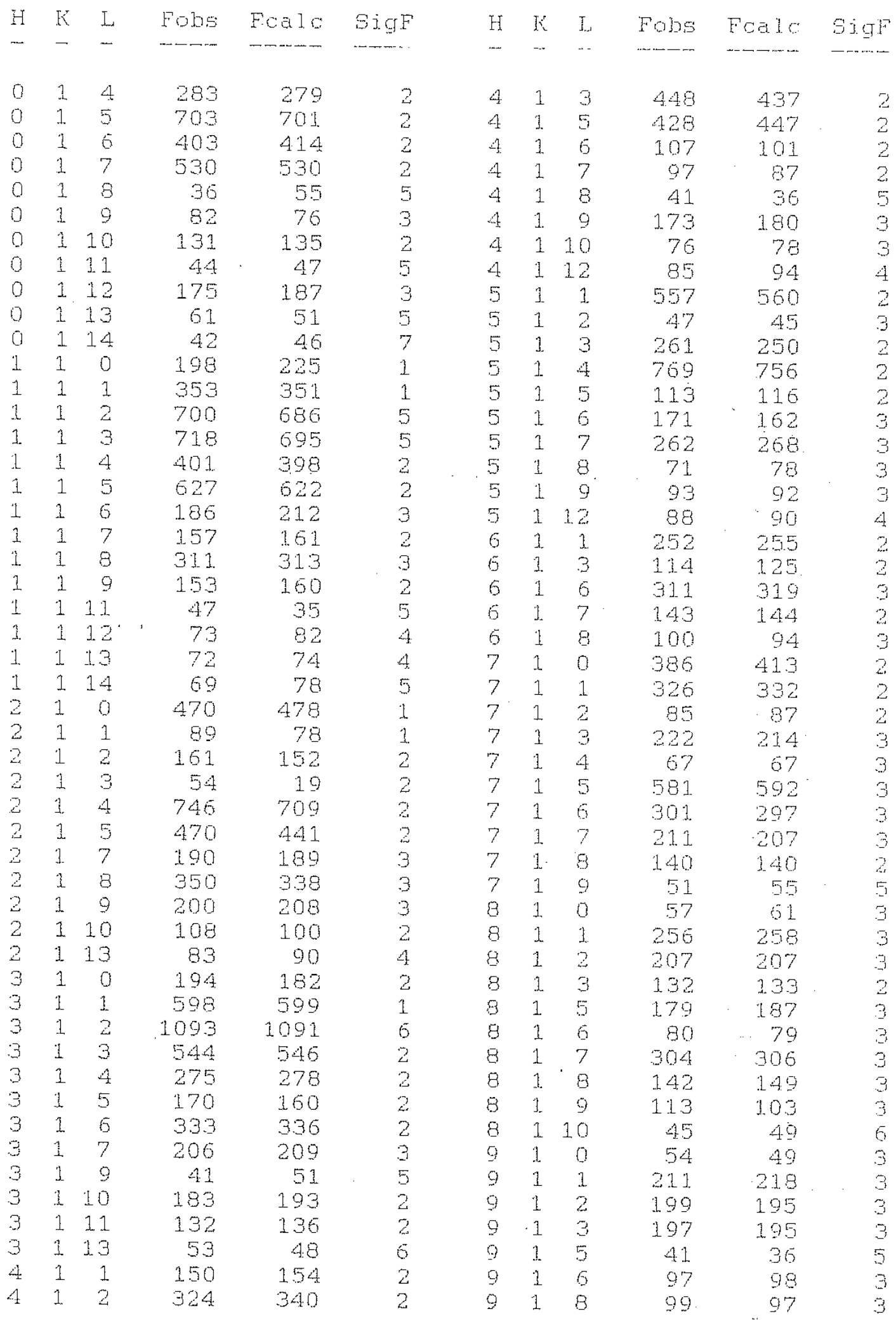




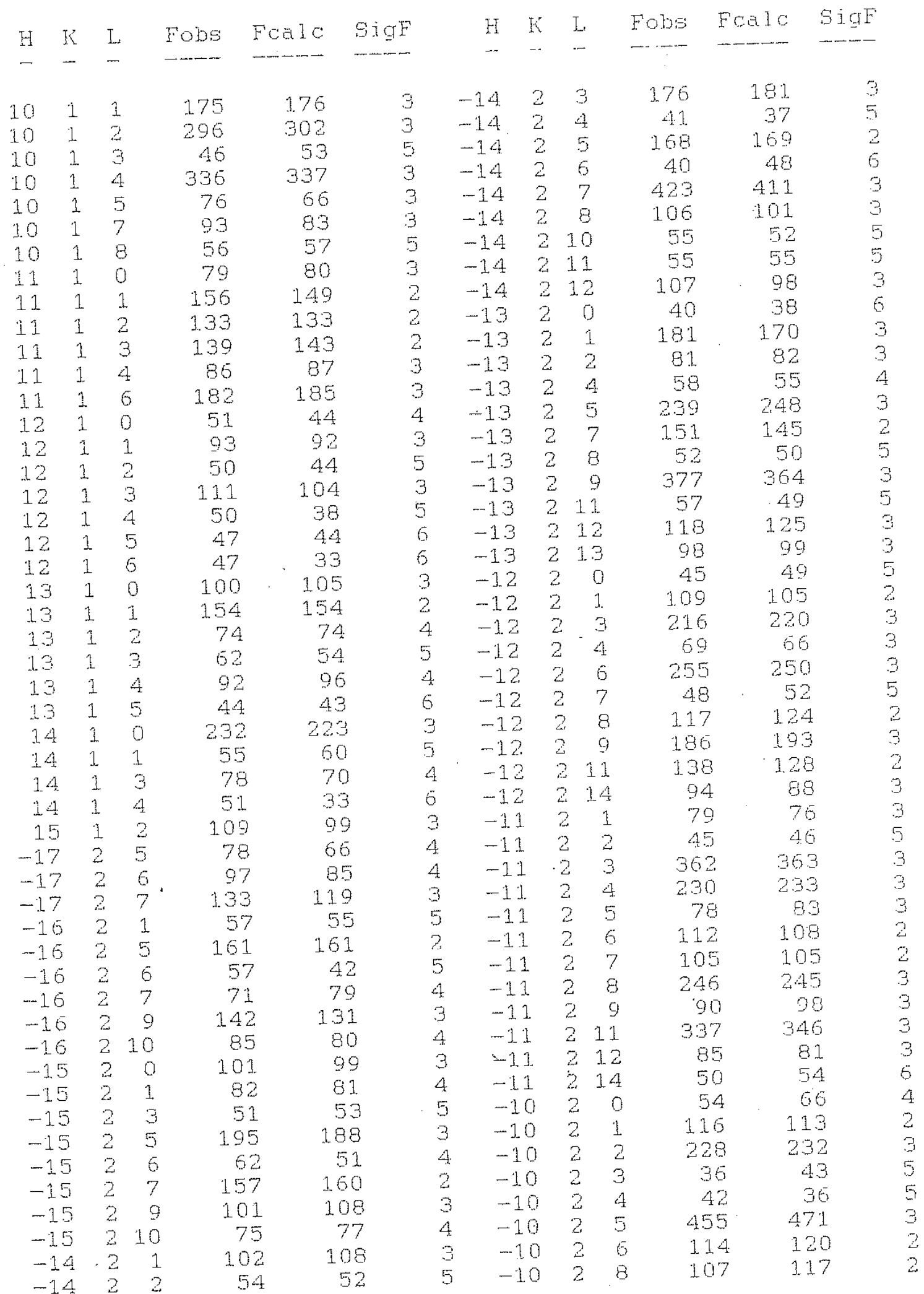




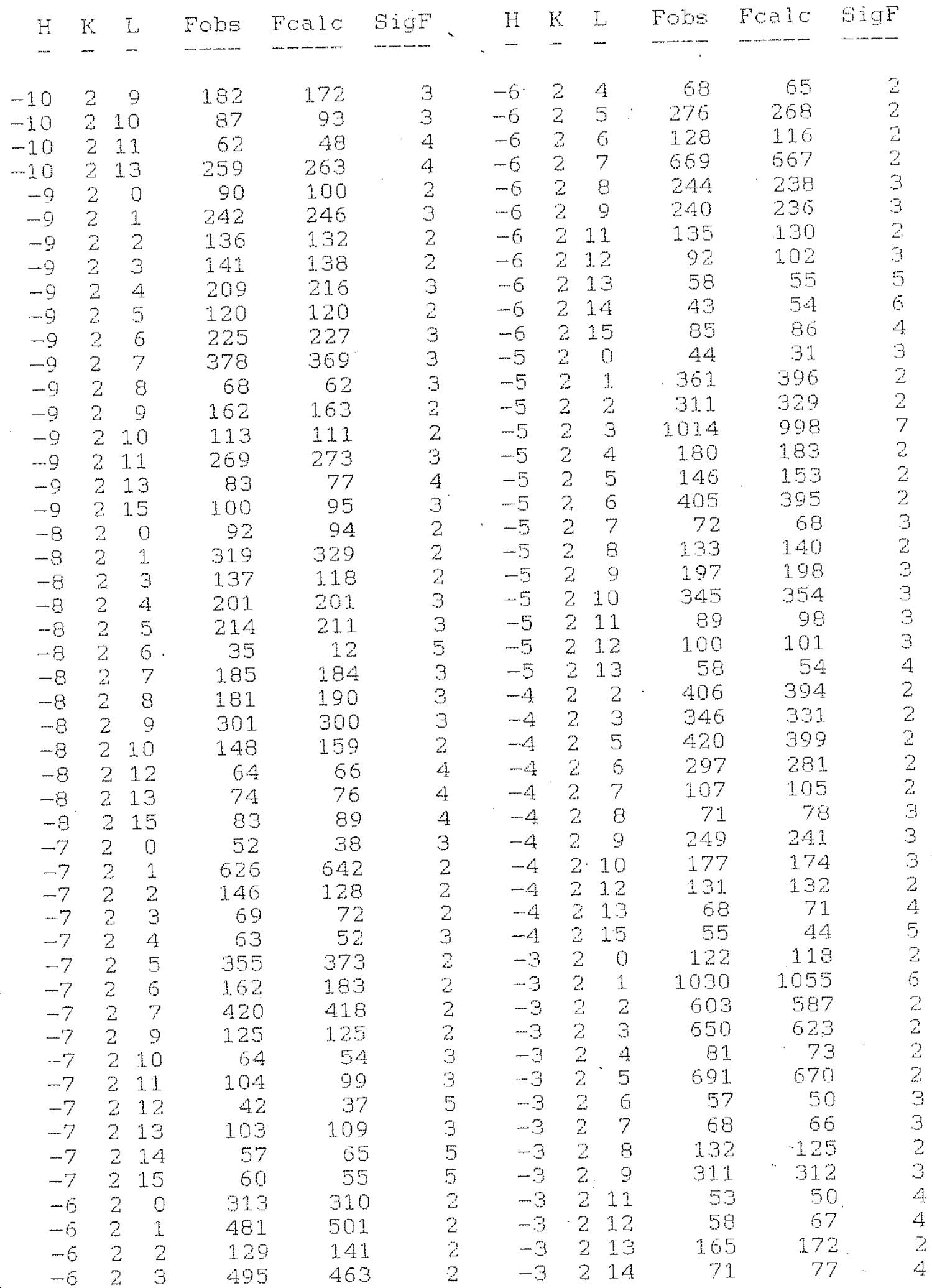


Vaiues of $10 \times$ Fobs and $10 \times \mathrm{FCal}$

Fage 8

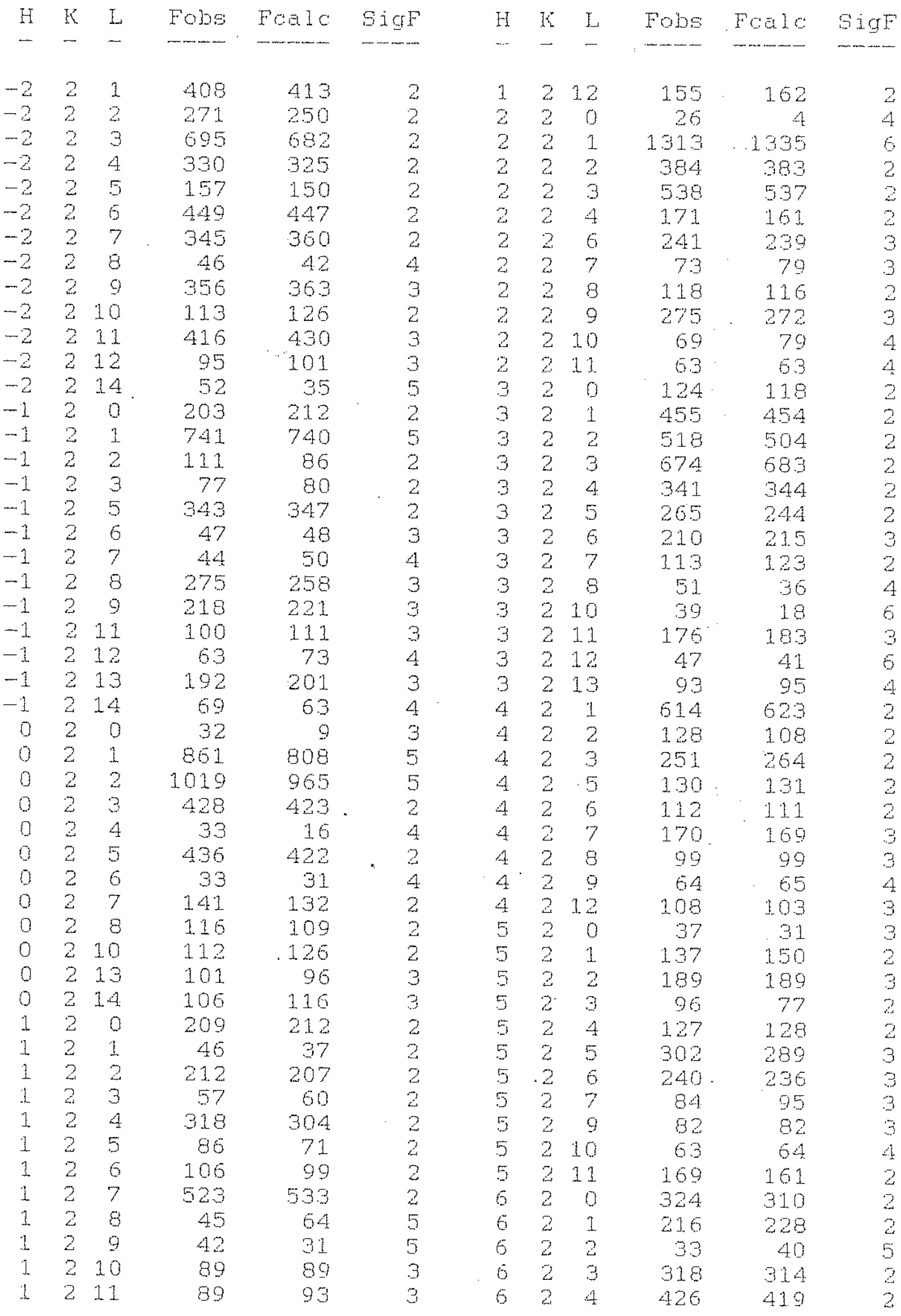


Values of $10 *$ Fobs and $10 *$ Fald

Page 9

\begin{tabular}{|c|c|c|c|c|c|c|c|c|c|c|}
\hline $\mathrm{H}$ & K L & Fobs & FCalc & SigF & $\mathrm{H}$ & K & $\stackrel{L}{-}$ & Fobs & Foalo & $\operatorname{sig} F$ \\
\hline 6 & 2 & 90 & 81 & 3 & 12 & 2 & & & & \\
\hline 6 & 2 & 109 & 108 & 2 & $\begin{array}{l}12 \\
12\end{array}$ & 2 & 6 & 76 & 77 & 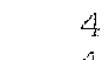 \\
\hline 8 & 2 & 213 & 219 & 3 & 13 & 2 & 2 & 80 & 70 & 4 \\
\hline 6 & 2 & $\begin{array}{r}88 \\
170\end{array}$ & & 3 & 13 & 2 & 3 & $\begin{array}{l}42 \\
96\end{array}$ & $\begin{array}{l}51 \\
90\end{array}$ & $\frac{6}{3}$ \\
\hline 6 & 210 & $\begin{array}{r}170 \\
74\end{array}$ & $\begin{array}{r}172 \\
72\end{array}$ & 2 & 13 & 2 & 4 & 87 & 80 & 4 \\
\hline 6 & 211 & 72 & $\begin{array}{l}72 \\
70\end{array}$ & 4 & 13 & 2 & 5 & 51 & 41 & \\
\hline 7 & 20 & 57 & $\begin{array}{l}70 \\
38\end{array}$ & 4 & 14 & 2 & 1 & 95 & 84 & 3 \\
\hline 7 & 2 & 289 & $\begin{array}{r}38 \\
293\end{array}$ & 3 & 14 & 2 & 2 & 72 & 82 & \\
\hline 7 & 3 & 313 & $\begin{array}{l}293 \\
319\end{array}$ & 3 & 14 & 2 & 3 & 166 & 148 & 3 \\
\hline 7 & 2 & 86 & $\begin{array}{r}119 \\
83\end{array}$ & 3 & 15 & 2 & 0 & 98 & 99 & 3 \\
\hline 7 & 2 & 1.41 & $\begin{array}{l}83 \\
137\end{array}$ & 3 & 15 & 2 & 1 & 98 & 91 & 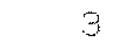 \\
\hline 7 & 2 & 126 & 120 & 2 & -16 & 3 & 2 & 43 & 41 & 7 \\
\hline 7 & 7 & 69 & 66 & 2 & -16 & 3 & 3 & 57 & 46 & 5 \\
\hline 7 & 8 & 77 & 79 & 4 & -16 & 3 & 4 & 75 & 67 & 4 \\
\hline 7 & 9 & 57 & 60 & $\begin{array}{l}3 \\
5\end{array}$ & -16 & 3 & 6 & 109 & 102 & 3 \\
\hline 8 & 2 & 100 & 94 & $\begin{array}{l}5 \\
2\end{array}$ & -16 & 3 & 7 & 121 & 119 & 3 \\
\hline 8 & 1 & 105 & 97 & $\begin{array}{l}2 \\
2\end{array}$ & -15 & 3 & 0 & 94 & 90 & 4 \\
\hline 8 & 2 & 165 & 149 & $\begin{array}{l}2 \\
3\end{array}$ & -15 & 3 & 1 & 92 & 94 & 4 \\
\hline 8 & 3 & 135 & 129 & $\begin{array}{l}3 \\
2\end{array}$ & -15 & 3 & 2 & 72 & 74 & 4 \\
\hline 8 & 4 & 80 & 81 & $\begin{array}{l}2 \\
3\end{array}$ & -15 & 3 & 3 & 148 & 143 & 3 \\
\hline 8 & 25 & 63 & 68 & 4 & -15 & 3 & 4 & 78 & 84 & 4 \\
\hline 8 & 6 & 171 & 170 & $\begin{array}{l}4 \\
3\end{array}$ & -15 & 3 & 5 & 92 & 83 & 3 \\
\hline 8 & 7 & 100 & 94 & 3 & $\begin{array}{l}-15 \\
-15\end{array}$ & 3 & 7 & 75 & 64 & 4 \\
\hline 8 & 8 & 62 & 56 & 5 & -15 & 3 & 9 & 1.24 & 124 & 3 \\
\hline 8 & 9 & 98 & 94 & 3 & -14 & 3 & 0 & 160 & 155 & 3 \\
\hline 9 & 0 & 95 & 100 & 2 & -14 & 3 & 1 & 134 & 136 & 3 \\
\hline 9 & 1 & 535 & 534 & 3 & -14 & 3 & 2 & 160 & 156 & 2 \\
\hline 9 & 2 & 110 & 108 & 2 & -14 & 3 & 3 & 72 & 77 & 4 \\
\hline 9 & 3 & 196 & 103 & 3 & -14 & 3 & 4 & 58 & 58 & 5 \\
\hline 9 & 4 & 128 & 126 & 2 & -14 & 3 & 5 & 53 & 51 & 5 \\
\hline 9 & 5 & 85 & 82 & 3 & -14 & 3 & 6 & 70 & 69 & 4 \\
\hline 9 & 2 & 133 & 133 & 3 & $\begin{array}{l}-14 \\
-14\end{array}$ & 3 & 7 & 113 & 115 & 3 \\
\hline 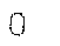 & 0 & 61 & 66 & 4 & $\begin{array}{l}-14 \\
-14\end{array}$ & 3 & 8 & 78 & 81 & 4 \\
\hline 0 & 1 & 1.88 & 185 & 3 & -14 & 3 & 9 & 1.43 & 135 & 3 \\
\hline 0 & 2 & 181 & 182 & 3 & -14 & 3 & 10 & 138 & 135 & 3 \\
\hline 10 & 3 & 268 & 259 & 3 & $\begin{array}{l}-13 \\
-13\end{array}$ & 3 & 0 & 143 & 140 & 2 \\
\hline 10 & 4 & 81 & 81 & 3 & $\begin{array}{l}-13 \\
-13\end{array}$ & 3 & 2 & 149 & 144 & 2 \\
\hline 10 & 5 & 112 & 104 & 3 & $\begin{array}{l}-13 \\
-13\end{array}$ & 3 & 3 & 150 & 1.61 & 2 \\
\hline 10 & 2 & 68 & 62 & 4 & $\begin{array}{l}-1.3 \\
-13\end{array}$ & 3 & 5 & 95 & 96 & 3 \\
\hline 10 & 7 & 118 & 111. & 3 & $\begin{array}{l}-13 \\
-13\end{array}$ & 3 & 6 & 43 & 45 & 5 \\
\hline 10 & 8 & 80 & 78 & 4 & $\begin{array}{l}-13 \\
-13\end{array}$ & 3 & 7 & 117 & 111 & 2 \\
\hline 11 & 2 & 98 & 104 & 3 & $\begin{array}{l}-13 \\
-13\end{array}$ & 3 & 10 & 182 & 174 & 3 \\
\hline 11 & 2 & 47 & 49 & 5 & $\begin{array}{l}-13 \\
-13\end{array}$ & 3 & 11 & 170 & 168 & 3 \\
\hline 11 & 3 & 41 & 44 & 5 & $\begin{array}{l}-13 \\
-12\end{array}$ & 3 & 12 & 180 & 167 & 3 \\
\hline 11 & 4 & 88 & 88 & 3 & $\begin{array}{l}-12 \\
-12\end{array}$ & 3 & 1 & 137 & 142 & 3 \\
\hline 11 & 5 & 70 & 58 & 4 & $\begin{array}{l}-12 \\
-12\end{array}$ & 3 & 2 & 129 & 127 & 2 \\
\hline 12 & 0 & 49 & 49 & 5 & $\begin{array}{l}-12 \\
-12\end{array}$ & 3 & 3 & 129 & 133 & 2 \\
\hline 12 & 2 & 63 & 55 & 4 & $\begin{array}{l}-12 \\
-12\end{array}$ & 3 & 4 & 84 & 90 & 3 \\
\hline 2 & 4 & 99 & 99 & 3 & $\begin{array}{l}-12 \\
-12\end{array}$ & $\begin{array}{l}3 \\
3\end{array}$ & 5 & $\begin{array}{r}115 \\
83\end{array}$ & 1.21 & 2 \\
\hline
\end{tabular}


Values of $10 *$ Fobs and $10 *$ Fealc

Page 10

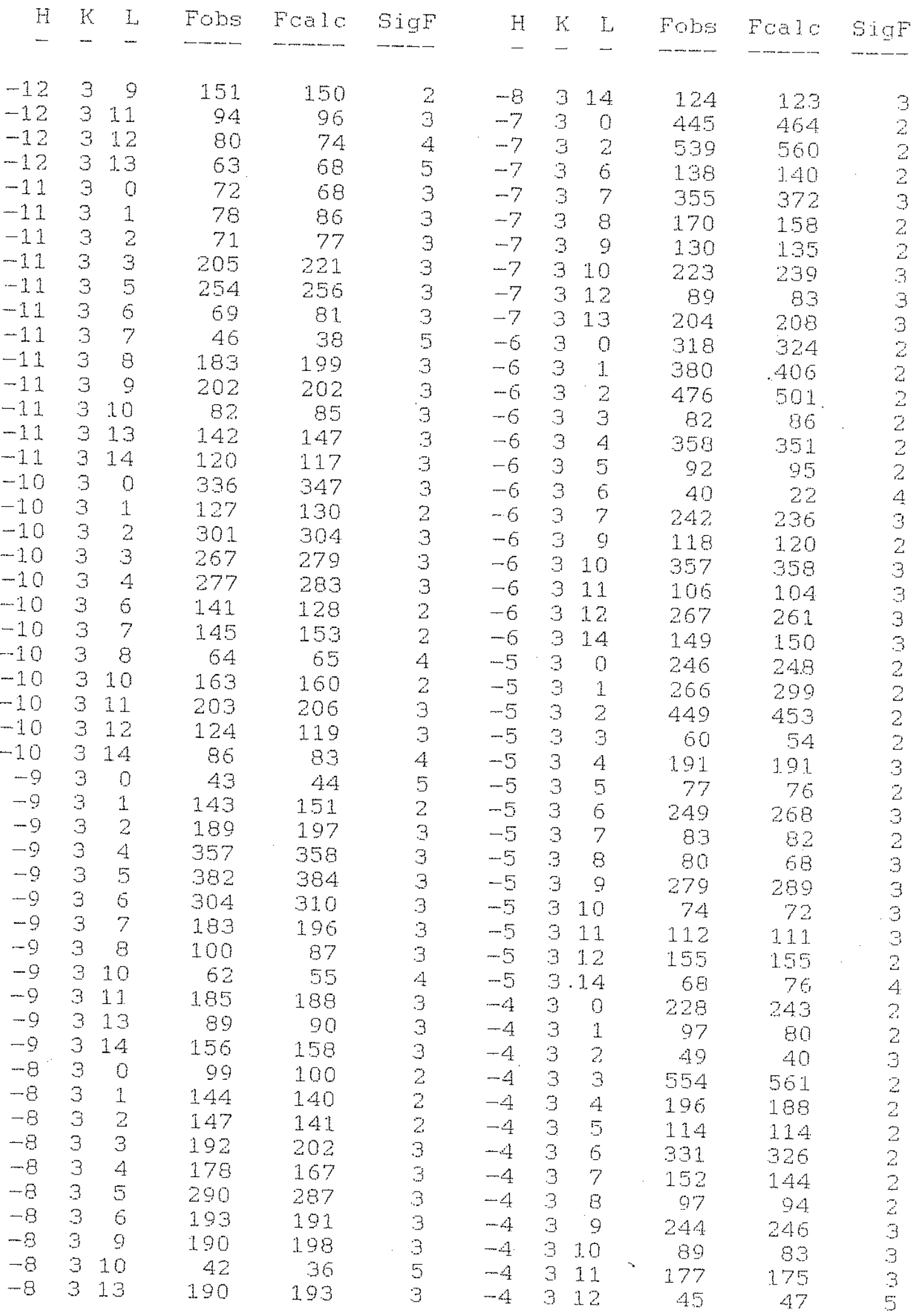


Values of $10 \times$ Fobs and $10 \times$ Fcalc

Page 11

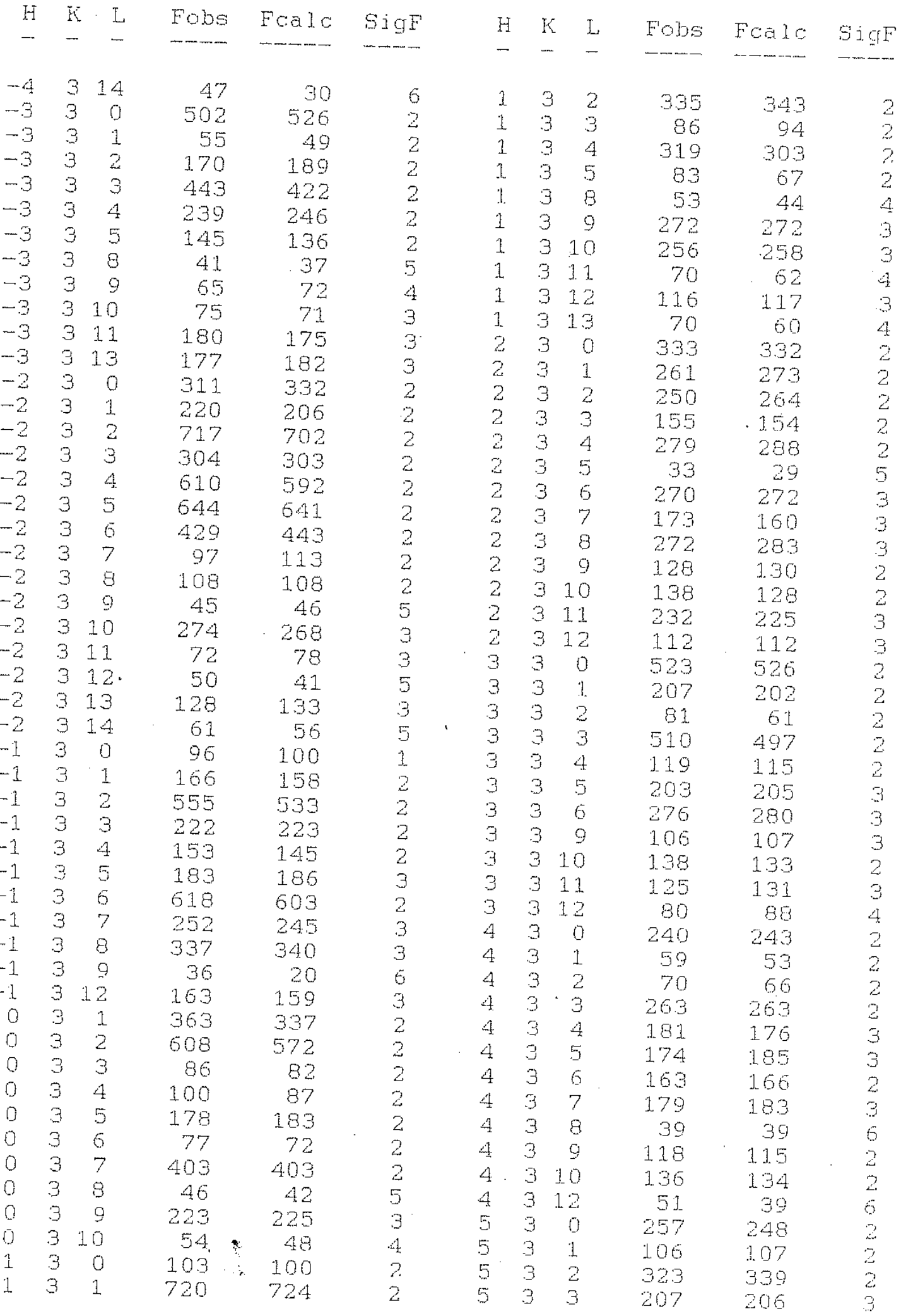


Values of $10 *$ Fobs and $10 \times$ Falo

Page 12

\begin{tabular}{|c|c|c|c|c|c|c|c|c|c|c|}
\hline $\mathrm{H}$ & $\begin{array}{ll}K & I \\
- & -\end{array}$ & Fobs & Fcalc & $\operatorname{SigF}$ & H & $\mathrm{K}$ & L & Fobs & Fcalc & SigF \\
\hline 5 & 4 & 359 & 364 & 3 & 11 & 3 & 0 & 73 & 68 & 3 \\
\hline 5 & 5 & 235 & 239 & 3 & 11 & 3 & 1 & 73 & 75 & 3 \\
\hline 5 & 3 & 284 & 294 & 3 & 11 & 3 & 2 & 131 & 1.33 & 2 \\
\hline 5 & 7 & 207 & 216 & 3 & 11 & 3 & 3 & 137 & 133 & 2 \\
\hline 5 & 8 & 147 & 143 & 2 & 11 & 3 & 4 & 108 & 101 & 3 \\
\hline 5 & 9 & 119 & 112 & 3 & 11 & 3 & 6 & 78 & 71 & 4 \\
\hline 5 & 310 & 46 & 37 & 6 & 12 & 3 & 0 & 37 & 30 & 6 \\
\hline 5 & 311 & 94 & 95 & 4 & 12 & 3 & 1 & 103 & 95 & 3 \\
\hline 6 & 3 & 334 & 324 & 2 & 12 & 3 & 2 & 70 & 74 & 4 \\
\hline 6 & 1 & 158 & 171 & 2 & 12 & 3 & 3 & 143 & 135 & 2 \\
\hline 6 & 2 & 255 & 254 & 3 & 12 & 3 & 4 & 66 & 61 & 4 \\
\hline 6 & 3 & 61 & 65 & 3 & 12 & 3 & 5 & 77 & 71 & 4 \\
\hline 6 & 4 & 52 & 64 & 4 & 13 & 3 & 0 & 1.48 & 140 & 2 \\
\hline 6 & 5 & 54 & 61 & 4 & 13 & 3 & 1 & 64 & 58 & 4 \\
\hline 6 & 3 & 310 & 296 & 3 & 14 & 3 & 0 & 159 & 1.55 & 2 \\
\hline 6 & 3 & 38 & 29 & 6 & 15 & 3 & 0 & 95 & 90 & 4 \\
\hline 6 & 8 & 176 & 173 & 3 & -15 & 4 & 2 & 84 & 78 & 4 \\
\hline 6 & 9 & 74 & 71 & 4 & -15 & 4 & 3 & 111 & 111 & 3 \\
\hline 6 & 310 & 46 & 31 & 6 & -15 & 4 & 7 & 87 & 76 & 4 \\
\hline 7 & 0 & 469 & 464 & 2 & -15 & 4 & 8 & 173 & 173 & 3 \\
\hline 7 & 3 & 314 & 327 & 3 & -14 & 4 & 2 & 128 & 125 & 3 \\
\hline 7 & 3 & 41 & 4.8 & 5 & -14 & 4 & 4 & 47 & 45 & 6 \\
\hline 7 & 3 & 186 & 191 & 3 & -14 & 4 & 5 & 194 & 192 & 3 \\
\hline 7 & 4 & 111 & 104 & 2 & -14 & 4 & 6 & 157 & 1.61 & 2 \\
\hline 7 & 5 & 161 & 163 & 2 & -14 & 4 & 8 & 131 & 135 & 3 \\
\hline 7 & 8 & 99 & 97 & 3 & -14 & 4 & 9 & 95 & 88 & 3 \\
\hline 7 & 9 & 81 & 75 & 4 & -14 & 41 & 0 & 71 & 65 & 4 \\
\hline 8 & 0 & 107 & 100 & 2 & -1.3 & 4 & 1 & 71 & 83 & 4 \\
\hline 8 & 1. & 126 & 128 & 2 & -13 & 4 & 2 & 126 & 124 & 3 \\
\hline $\mathrm{B}$ & 2 & 45 & 42 & 5 & -13 & 4 & 5 & 93 & 88 & 3 \\
\hline 8 & 3 & 299 & 291 & 3 & -13 & 4 & 6 & 67 & 70 & 4 \\
\hline 8 & 5 & 46 & 41 & 5 & -13 & 4 & 7 & 234 & 232 & 3 \\
\hline 8 & 6 & 58 & 55 & 4 & -13 & 4 & 8 & 329 & 137 & 3 \\
\hline 8 & 7 & 118 & 114 & 3 & -13 & 4 & 9 & 46 & 66 & 6 \\
\hline 8 & 8 & 77 & 80 & 4 & -13 & 41 & 0 & 139 & 139 & 3 \\
\hline$\theta$ & 0 & 42 & 44 & 5 & -13 & 41 & 1. & 125 & 123 & 3 \\
\hline 9 & 1 & 116 & 1.15 & 2 & -12 & 4 & 0 & 66 & 57 & 4 \\
\hline 9 & 2 & 226 & 230 & 3 & -12 & 4 & 1 & 51 & 59 & 5 \\
\hline 9 & 3 & 70 & 67 & 3 & -12 & 4 & 3. & 109 & 105 & 3 \\
\hline 9 & 4 & 187 & 182 & 3 & -12 & 4 & 4 & 172 & 171 & 2 \\
\hline 9 & 5 & 121 & 116 & 2 & -12 & 4 & 5 & 67 & 61 & 4 \\
\hline 9 & 3 & 67 & 63 & 4 & -12 & 4 & 6 & 116 & 114 & 2 \\
\hline 9 & 3 & 79 & 83 & 1 & -12 & 4 & 7 & 88 & 84 & 3 \\
\hline 9 & 8 & 45 & 39 & 6 & -12 & 4 & 8 & 184 & 183 & 3 \\
\hline 10 & 0 & 347 & 347 & 3 & -12 & 4 & 9 & 161 & 159 & 2 \\
\hline 10 & 1 & 180 & 174 & 2 & -12 & 41 & 0 & 52 & 55 & 5 \\
\hline 10 & 2 & 72 & 76 & 3 & -12 & 41 & 1 & 144 & 150 & 3 \\
\hline 10 & 4 & 138 & 138 & 2 & -12 & 41 & 2 & 122 & 122 & 3 \\
\hline 10 & 5 & 144 & 140 & 2 & -11 & 4 & 1. & 59 & 54 & 4 \\
\hline 10 & 0 & 248 & 245 & 3 & -11 & 4 & 2 & 148 & 149 & 2 \\
\hline
\end{tabular}


Values of $10 *$ Fobs and $10 *$ Fala

Page 13

\begin{tabular}{|c|c|c|c|c|c|c|c|c|c|c|c|}
\hline $\mathrm{H}$ & $\mathrm{K}$ & L & Fobs & $\begin{array}{l}F c a l c \\
-\end{array}$ & $S i g F$ & H & $\begin{array}{l}\mathrm{K} \\
-\end{array}$ & 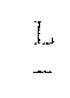 & Fobs & Fode & Sigh \\
\hline-11 & 4 & 3 & 79 & 79 & 3 & -7 & 4 & 14 & 48 & 51 & 6 \\
\hline-1.1 & 4 & 4 & 69 & 61 & 3 & -6 & 4 & 0 & 165 & 176 & 2 \\
\hline-11 & 4 & 5 & 67 & 73 & 4 & -6 & 4 & 1 & 144 & 157 & 2 \\
\hline-11 & 4 & 6 & 168 & 162 & 2 & -6 & 4 & 2 & 42 & 51 & 4 \\
\hline-11 & 4 & 8 & 95 & 88 & 3 & -6 & 4 & 3 & 173 & 170 & 3 \\
\hline-11 & 4 & 9 & 47 & 36 & 5 & -6 & 4 & 4 & 264 & 266 & 3 \\
\hline-11 & 4 & 10 & 214 & 215 & 3 & -6 & 4 & 5 & 347 & 350 & 3 \\
\hline-11 & 4 & 11 & 78 & 72 & 4 & -6 & 4 & 7 & 135 & 136 & 2 \\
\hline-11 & 4 & 12 & 124 & 130 & 3 & -6 & 4 & 8 & 198 & 200 & 3 \\
\hline-11 & 4 & 13 & 138 & 140 & 3 & -6 & 4 & 10 & 112 & 120 & 2 \\
\hline-10 & 4 & 3 & 188 & 199 & 3 & -6 & 4 & 11 & 86 & 77 & 3 \\
\hline-10 & 4 & 7 & 218 & 220 & 3 & -6 & 4 & 13 & 57 & 56 & 5 \\
\hline-10 & 4 & 8 & 94 & 95 & 3 & -5 & 4 & 0 & 176 & 191 & 3 \\
\hline-10 & 4 & 10 & 96 & 94 & 3 & -5 & 4 & 1 & 164 & 180 & 3 \\
\hline-10 & 4 & 11 & 1.16 & 115 & 3 & -5 & 4 & 2 & 386 & 396 & 2 \\
\hline-10 & 4 & 12 & 132 & 125 & 3 & -5 & 4 & 3 & 109 & 107 & 2 \\
\hline-10 & 4 & 13 & 79 & 78 & 4 & -5 & 4 & 4 & 200 & 197 & 3 \\
\hline-9 & 4 & 0 & 227 & 234 & 3 & -5 & 4 & 5 & 358 & 366 & 3 \\
\hline-9 & 4 & 1 & 113 & 108 & 2 & -5 & 4 & 6 & 151 & 150 & ? \\
\hline-9 & 4 & 3 & 115 & 120 & 2 & -.5 & 4 & 7 & 370 & 370 & 3 \\
\hline-9 & 4 & 4 & 136 & 132 & 2 & -5 & 4 & 9 & 106 & 119 & 2 \\
\hline-9 & 4 & 5 & 380 & 380 & 3 & -5 & 4 & 10 & 63 & 59 & 4 \\
\hline-9 & 4 & 6 & 156 & 158 & 2 & -5 & 4 & 11 & 98 & 100 & 3 \\
\hline-9 & 4 & 8 & 119 & 113 & 2 & -5 & 4 & 12 & 49 & 53 & 5 \\
\hline-9 & 4 & 9 & 213 & 214 & 3 & -4 & 4 & 1 & 114 & 124 & 2 \\
\hline-9 & 4 & 12 & 199 & 209 & 3 & -4 & 4 & 2 & 470 & 484 & 2 \\
\hline-9 & 4 & 13 & 131 & 130 & 3 & -4 & 4 & 3 & 111 & 96 & 2 \\
\hline-8 & 4 & 0 & 165 & 181 & 2 & -4 & 4 & 4 & 251 & 255 & 3 \\
\hline-8 & 4 & 1 & 175 & 189 & 3 & -4 & 4 & 5 & 154 & 158 & 3 \\
\hline-8 & 4 & 2 & 61 & 61 & 3 & -4 & 4 & 6 & 388 & 372 & 3 \\
\hline-8 & 4 & 3 & 106 & 112 & 2 & -4 & 4 & 7 & 93 & 94 & 3 \\
\hline-8 & 4 & 5 & 97 & 109 & 3 & -4 & 4 & 8 & 348 & 355 & 3 \\
\hline-8 & 4 & 6 & 249 & 24.5 & 3 & -4 & 4 & 9. & 219 & 226 & 3 \\
\hline-8 & 4 & 7 & 353 & 353 & 3 & -4 & 4 & 10 & 172 & 178 & 2 \\
\hline-8 & 4 & 9 & 123 & 125 & 2 & -4 & 4 & 14 & 43 & 43 & 7 \\
\hline-8 & 4 & 11 & 193 & 182 & 3 & -3 & 4 & 0 & 178 & 190 & 3 \\
\hline-7 & 4 & 0 & 308 & 322 & 3 & -3 & 4 & 1 & 124 & 128 & 2 \\
\hline-7 & 4 & 1. & 196 & 214 & 3 & -3 & 4 & 2 & 117 & 124 & 2 \\
\hline-7 & 4 & 2 & 471 & 492 & 2 & -3 & 4 & 3 & 102 & 104 & 2 \\
\hline-7 & 4 & 3 & 94 & 103 & 2 & -3 & 4 & 4 & 439 & 443 & 2 \\
\hline-7 & 4 & 4 & 107 & 98 & 2 & -3 & 4 & 5 & 194 & 184 & $\exists$ \\
\hline-7 & 4 & 5 & 45 & 23 & 4 & -3 & 4 & 6 & 69 & 67 & 3 \\
\hline-7 & 4 & 6 & 131 & 124 & 2 & -3 & 4 & 7 & 471 & 458 & 3 \\
\hline-7 & 4 & 7 & 41 & 38 & 5 & -3 & 4 & 8 & 135 & 131 & 2 \\
\hline-7 & 4 & 8 & 175 & 171 & 3 & -3 & 4 & 9 & 90 & 92 & 3 \\
\hline-7 & 4 & 9 & 209 & 210 & 3 & -3 & 4 & 10 & 95 & 105 & 3 \\
\hline-7 & 4 & 10 & 41 & 54 & 5 & -3 & 4 & 11 & 69 & 69 & 4 \\
\hline-7 & 4 & 11 & 44 & 49 & 5 & -3 & 4 & 12 & 169 & 164 & 3 \\
\hline-7 & 4 & 12 & 101 & 94 & 3 & -2 & 4 & 0 & 91. & 103 & 2 \\
\hline-7 & 4 & 13 & 107 & 115 & 3 & -2 & 4 & 1 & 716 & 748 & 2 \\
\hline
\end{tabular}


Values of 10*Fobs and 10*Foalc

Pare 14

\begin{tabular}{|c|c|c|c|c|c|c|c|c|c|}
\hline $\mathrm{K}$ & Fobs & $F \operatorname{calc}$ & SigF & $\mathrm{H}$ & $\mathrm{K}$ & $\mathrm{L}$ & Fobs & Foalc & SigF \\
\hline- & $-\cdots$ & $---1-$ & $-m-$ & - & - & - & $--m$ & $--\infty-\cdots$ & $-\cdots$ \\
\hline 4 & 88 & 63 & 2 & 2 & 4 & 4 & 174 & 159 & 3 \\
\hline 4 & 83 & 74 & 2 & 2 & 4 & 5 & 202 & 209 & 3 \\
\hline 5 & 47 & 50 & 4 & 2 & 4 & 6 & 157 & 151 & 2 \\
\hline 4 & 108 & 96 & 2 & 2 & 4 & 7 & 294 & 294 & 3 \\
\hline 4 & 228 & 232 & 3 & 2 & 4 & 9 & 48 & 57 & 5 \\
\hline 4 & 288 & 293 & 3 & 2 & 4 & 10 & 42 & 39 & 6 \\
\hline 410 & 155 & 151 & 2 & 2 & 4 & 12 & 41 & 27 & 7 \\
\hline 411 & 72 & 75 & 4 & 3 & 4 & 0 & 196 & 190 & 3 \\
\hline $4 \quad 12$ & 108 & 109 & 3 & 3 & 4 & 1. & 617 & 614 & 2 \\
\hline 4 & 95 & 83 & 2 & 3 & 4 & 2 & 79 & 70 & 2 \\
\hline 4 & 222 & 214 & 2 & 3 & 4 & 3 & 42 & 47 & 4 \\
\hline 2 & 233 & 236 & 2 & 3 & 4 & 4 & 231 & 225 & 3 \\
\hline 4 & 415 & 419 & 2 & 3 & 4 & 5 & 193 & 185 & 3 \\
\hline 4 & 78 & 82 & 2 & 3 & 4 & 6 & 188 & 190 & 3 \\
\hline 5 & 297 & 288 & 3 & 3 & 4 & 8 & 210 & 203 & 3 \\
\hline 6 & 402 & 389 & 3 & 3 & 4 & 9 & 296 & 300 & 3 \\
\hline 4 & 53 & 55 & 4 & 3 & 4 & 10 & 105 & 107 & 3 \\
\hline 4 & 151 & 151 & 2 & 3 & 4 & 11 & 82 & 87 & 4 \\
\hline 410 & 52 & 50 & 5 & 4 & 4 & 0 & 37 & 24 & 4 \\
\hline 411 & 156 & 156 & 2 & 4 & 4 & 1 & 129 & 126 & 2 \\
\hline 412 & 83 & $B 1$ & 4 & 4 & 4 & 2 & 378 & 375 & 2 \\
\hline 4 & 549 & 559 & 2 & 4 & 4 & 3 & 397 & 403 & 3 \\
\hline 4 & 255 & 238 & 2 & 4 & 4 & 4 & 121 & 135 & 2 \\
\hline 4 & 363 & 360 & 2 & 4 & 4 & 6 & 99 & 102 & 3 \\
\hline 4 & 82 & 76 & 2 & 4 & 4 & 8 & 103 & 101 & 3 \\
\hline 4 & 92 & 90 & 2 & 4 & 4 & 11 & 109 & 108 & 3 \\
\hline 4 & 135 & 130 & 2 & 5 & 4 & 0 & 196 & 191 & 3 \\
\hline 4 & 349 & 338 & 3 & 5 & 4 & 1 & 332 & 339 & 3 \\
\hline 4 & 262 & 255 & 3 & 5 & 4 & 2 & 231 & 228 & 3 \\
\hline 49 & 119 & 126 & 2 & 5 & 4 & 4 & 336 & 336 & 3 \\
\hline 410 & 155 & 166 & 2 & 5 & 4 & 5 & 107 & 104 & 2 \\
\hline $4 \quad 12$ & 139 & 126 & 3 & 5 & 4 & 6 & 76 & 84 & 3 \\
\hline 413 & 46 & 56 & 6 & 5 & 4 & 8 & 53 & 51 & 5 \\
\hline 4 & 98 & 83 & 2 & 5 & 4 & 9 & 40 & 34 & 6 \\
\hline 4 & 158 & 185 & 2 & 6 & 4 & 0 & 176 & 176 & 2 \\
\hline 4 & 449 & 442 & 2 & 6 & 4 & 1. & 346 & 354 & 3 \\
\hline 4 & 166 & 164 & 3 & $\theta$ & 4 & 3 & 216 & 217 & 3 \\
\hline 4 & 112 & 123 & 2 & 6 & 4 & 5 & 151 & 346 & 2 \\
\hline 4 & 125 & 119 & 2 & 6 & 4 & 7 & 53 & 49 & 5 \\
\hline 6 & 151 & 146 & 2 & 6 & 4 & .8 & 97 & 90 & 3 \\
\hline 7 & 66 & 56 & 3 & 6 & 4 & 10 & 46 & 34 & 6 \\
\hline 8 & 124 & 119 & 2 & 7 & 4 & 0 & 322 & 322 & 3 \\
\hline 4 & 129 & 134 & 2 & 7 & 4 & 1 & 39 & 42 & 5 \\
\hline 410 & 93 & 98 & 3 & 7 & 4 & 2 & 126 & 126 & 2 \\
\hline 411 & 66 & 61 & 4 & 7 & 4 & 3 & 148 & 148 & 2 \\
\hline 412 & 98 & 102 & 3 & 7 & 4 & 4 & 80 & 84 & 3 \\
\hline 40 & 97 & 103 & 2 & 7 & $A$ & 5 & 157 & 160 & 2 \\
\hline 1 & 49 & 55 & 3 & 7 & 4 & 6 & 72 & .75 & 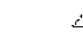 \\
\hline 2 & 279 & 292 & 2 & 7 & 4 & 7 & 124 & 120 & \\
\hline 43 & 202 & 192 & 3 & 7 & 4 & 8 & 83 & 77 & 4 \\
\hline
\end{tabular}


Values of $10 *$ Fobs and $10 * \mathrm{FCa} 0$

Page 15

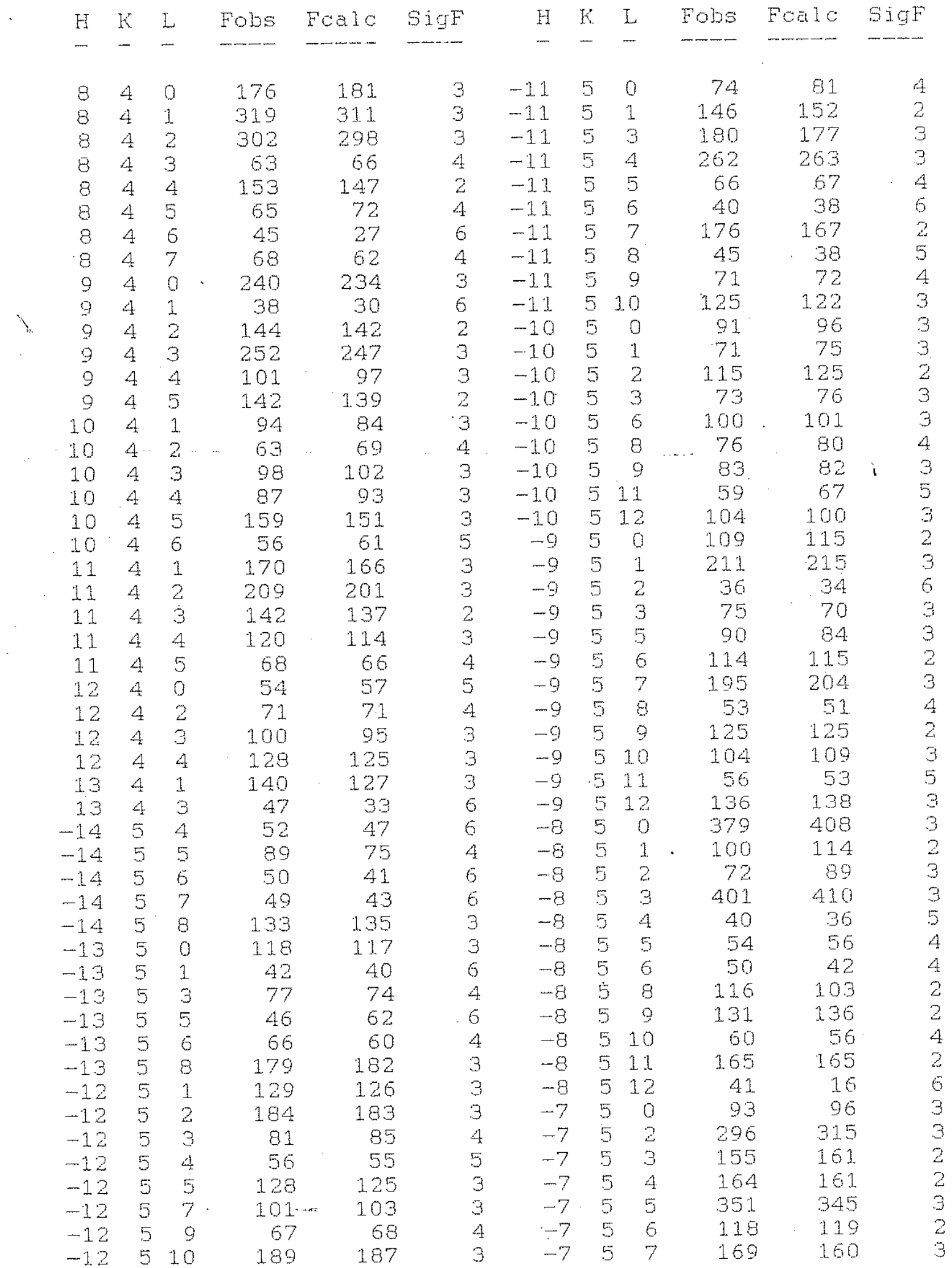




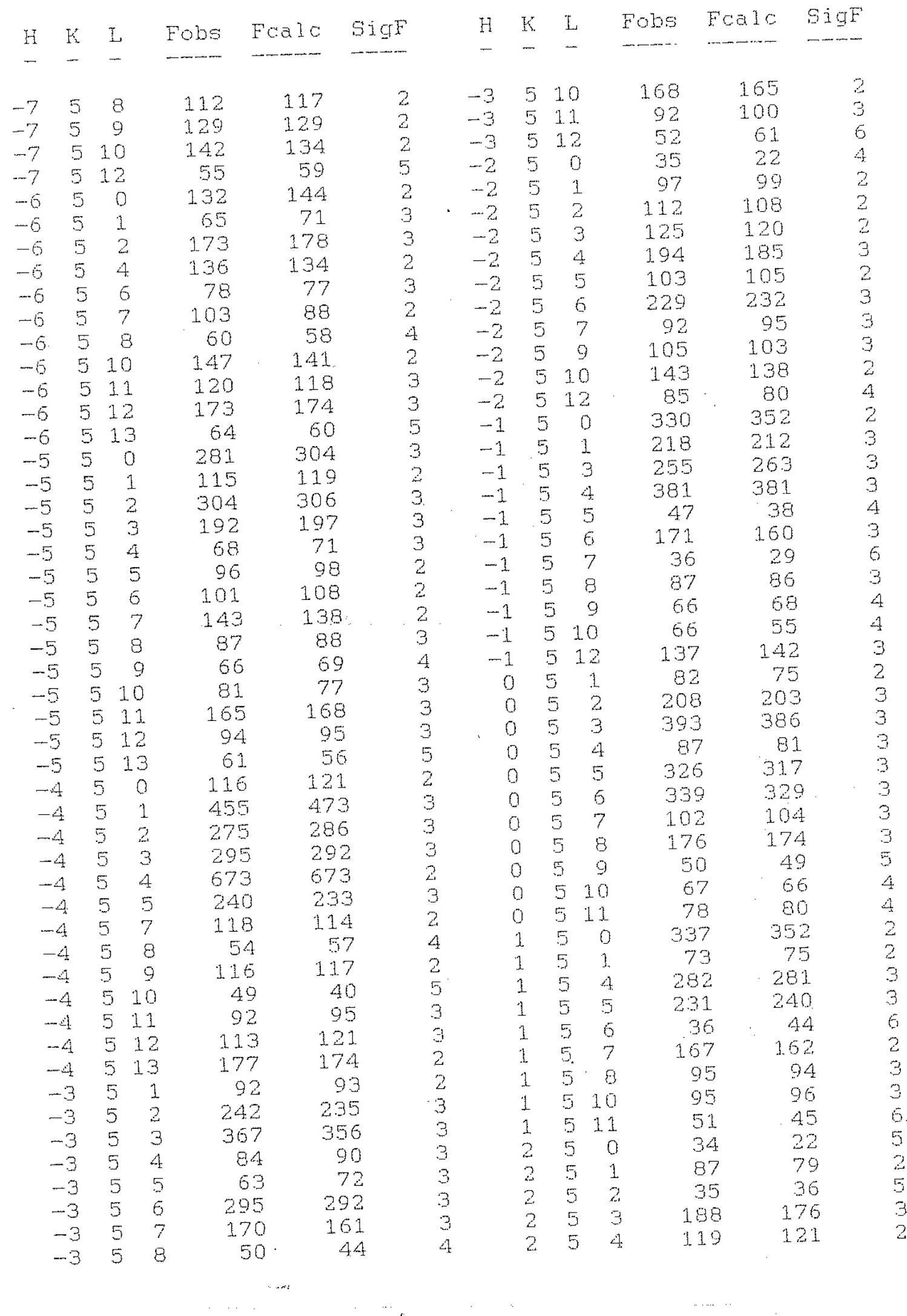


Values of $10 *$ Fobs and $10 *$ Foalc

Page 17

\begin{tabular}{|c|c|c|c|c|c|c|c|c|c|c|}
\hline $\mathrm{H}$ & K L & Fobs & Fcalc & $\operatorname{SigF}$ & $\mathrm{H}$ & K & L & Fobs & Foalc & $S i g F$ \\
\hline- & - & & - & - & - & - & - & $\rightarrow$ & 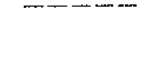 & \\
\hline 2 & 5 & 41 & 46 & 5 & 8 & 5 & 5 & 175 & 172 & 3 \\
\hline 2 & 6 & 162 & 162 & 2 & 8 & 5 & 6 & 98 & 96 & 3 \\
\hline 2 & 7 & 71 & 72 & 3 & 8 & 5 & 7 & 119 & 103 & 3 \\
\hline 2 & 8 & 131 & 127 & 2 & 9 & 5 & 0 & 116 & 115 & 2 \\
\hline$\dot{L}$ & 510 & 121 & 117 & 3 & 9 & 5 & 1 & 60 & 65 & 4 \\
\hline 2 & 511 & 68 & 67 & 5 & 9 & 5 & 2 & 163 & 161 & 2 \\
\hline 3 & 1 & 224 & 227 & 3 & 9 & 5 & 4 & 83 & 82 & 4 \\
\hline 3 & 2 & 121 & 120 & 2 & 10 & 5 & 0 & 90 & 96 & 3 \\
\hline 3 & 3 & 185 & 195 & 3 & 10 & 5 & 1 & 61 & 55 & 5 \\
\hline 3 & 4 & 208 & 206 & 3 & 10 & 5 & 2 & 84 & 77 & 4 \\
\hline 3 & 5 & 208 & 194 & 3 & 11 & 5 & 0 & 80 & 81 & 4 \\
\hline 3 & 6 & 110 & 110 & 2 & 1.1 & 5 & 2 & 134 & 127 & 3 \\
\hline 3 & 8 & 99 & 92 & 3 & 11 & 5 & 3 & 58 & 50 & 5 \\
\hline 3 & 510 & 110 & 102 & 3 & 11 & 5 & 4 & 68 & 67 & 5 \\
\hline 4 & 5 & 124 & 121 & 2 & 12 & 5 & 1 & 130 & 129 & 3 \\
\hline 4 & 1 & 131 & 130 & 2 & 13 & 5 & 0 & 125 & 117 & 3 \\
\hline 4 & 5 & 62 & 57 & 3 & 13 & 5 & 1 & 56 & 47 & 5 \\
\hline 4 & 3 & 168 & 173 & 3 & -12 & 6 & 1 & 58 & 51 & 5 \\
\hline 4 & 4 & 193 & 191 & 3 & -12 & 6 & 2 & 73 & 78 & 4 \\
\hline 4 & 5 & 94 & 95 & 3 & -12 & 6 & 3 & 70 & 71 & 4 \\
\hline 4 & 5 & 89 & 81 & 3 & -12 & 6 & 4 & 99 & 110 & 3 \\
\hline 4 & 7 & 180 & 174 & 3 & -12 & 6 & 5 & 126 & 128 & 3 \\
\hline 4 & 5 & 106 & 98 & 3 & -12 & 6 & 6 & 175 & 172 & 3 \\
\hline 4 & 510 & 64 & 63 & 5 & -1.2 & 6 & 7 & 46 & 35 & 6 \\
\hline 5 & 50 & 303 & 304 & 3 & -12 & 6 & 8 & 85 & 89 & 4 \\
\hline 5 & 1 & 282 & $284^{\circ}$ & 3 & -11 & 6 & 0 & 171 & 170 & 2 \\
\hline 5 & 3 & 52 & 64 & 4 & -11 & 6 & 1 & 99 & 104 & 3 \\
\hline 5 & 4 & 120 & 119 & 2 & -11 & 6 & 3 & 92 & 91 & 3 \\
\hline 5 & 5 & 38 & 40 & 6 & -11 & 6 & 4 & 102 & 106 & 3 \\
\hline 5 & 6 & 272 & 266 & 3 & -11 & 6 & 6 & 83 & 90 & 4 \\
\hline 5 & 8 & 71 & 70 & 4 & -11 & 6 & 7 & 83 & 81 & 4 \\
\hline 5 & 9 & 161 & 158 & 2 & -11 & 6 & 8 & 240 & 229 & 3 \\
\hline 6 & 0 & 139 & 144 & 2 & -11 & 6 & 9 & 122 & 117 & 3 \\
\hline 6 & 5 & 134 & 136 & 2 & -10 & 5 & 1. & 53 & 54 & 5 \\
\hline 6 & 5 & 214 & 214 & 3 & -10 & 6 & 2 & 60 & 61 & 4 \\
\hline$E$ & 5 & 78 & 78 & 3 & -10 & 6 & 4 & 45 & 43 & 5 \\
\hline 6 & 5 & 79 & 79 & 3 & -10 & 6 & 5 & 119 & 1.13 & 3 \\
\hline 6 & 5 & 69 & 72 & 4 & -10 & 6 & 6 & 79 & 89 & 4 \\
\hline 6 & 5 & 160 & 159 & 3 & -10 & 6 & 7 & 68 & 64 & 4 \\
\hline 7 & 5 & 101 & 96 & 3 & -10 & 6 & 10 & 240 & 222 & 3 \\
\hline 7 & 5 & 50 & 51 & 5 & -9 & 6 & 0 & 140 & 144 & 2 \\
\hline 7 & 5 & 92 & 93 & 3 & -9 & 6 & 1 & 139 & 150 & 0 \\
\hline 7 & 5 & 230 & 228 & 3 & -9 & .6 & 2 & 104 & 110 & $\cong$ \\
\hline 7 & 4 & 54 & 51 & 4 & -9 & 6 & 3 & 175 & 179 & 3 \\
\hline 7 & 5 & 142 & 142 & 2 & -9 & 6 & 5 & 172 & 171 & 3 \\
\hline 7 & 5 & 110 & 105 & 3 & -9 & 6 & 6 & 66 & 59 & \\
\hline 7 & 5 & 120 & 122 & 3 & -9 & 6 & 7 & 49 & 52 & \\
\hline 7 & 8 & 72 & 74 & 4 & -9 & 6 & 8 & 115 & 111 & \\
\hline 8 & 0 & 408 & 408 & 3 & -9 & 6 & 9 & 114 & 109 & \\
\hline 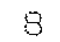 & 4 & 111 & 104 & 3 & -9 & 6 & 10 & 160 & 165 & \\
\hline
\end{tabular}


Values of $10 \times$ Fobs and $10 *$ Fald

Page 18

\begin{tabular}{|c|c|c|c|c|c|c|c|c|c|c|c|}
\hline $\mathrm{H}$ & K & 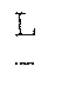 & Fobs & Foalc & $\operatorname{SigF}$ & $\mathrm{H}$ & $K$ & $\mathrm{~L}_{\mathrm{r}}$ & Fobs: & Fcalc & $S i g F$ \\
\hline 8 & 6 & 0 & 52 & 52 & 5 & -4 & 6 & 11 & 70 & 73 & 4 \\
\hline 8 & 6 & 1 & 138 & 144 & 2 & -3 & 6 & 0 & 152 & 168 & 2 \\
\hline-8 & 6 & 2 & 76 & 76 & 3 & -3 & 6 & 1 & 126 & 130 & 2 \\
\hline-8 & 6 & 3 & 58 & 55 & 4 & -3 & 6 & 2 & 177 & 175 & 3 \\
\hline-8 & 6 & 4 & 143 & 153 & 2 & -3 & 6 & 3 & 59 & 51 & 3 \\
\hline-8 & 6 & 5 & 140 & 141 & 2 & -3 & 6 & 4 & 210 & 210 & \\
\hline-8 & 6 & 6 & 81 & 80 & 3 & -3 & 6 & 6 & 47 & 45 & \\
\hline-8 & 6 & 7 & 100 & 98 & 3 & -3 & 6 & 7 & 65 & 60 & \\
\hline-8 & 6 & 8 & 153 & 151 & 2 & -3 & 6 & 8 & 61 & 67 & \\
\hline-8 & 6 & 9 & 54 & 56 & 5 & -3 & 6 & 9 & 78 & 71 & \\
\hline-8 & 6 & 10 & 70 & 62 & 4 & -3 & 6 & 10 & 124 & 116 & \\
\hline-8 & 6 & 11 & 46 & 48 & 6 & -3 & 6 & 11 & 57 & 51 & \\
\hline-7 & 6 & 0 & 109 & 116 & 2 & -2 & 6 & 0 & 77 & 75 & \\
\hline-7 & 6 & 1 & 121 & 124 & 2 & -2 & 6 & 2 & 41 & 46 & \\
\hline-7 & 6 & 2 & 110 & 114 & 2 & -2 & 6 & 3 & 52 & 51 & \\
\hline-7 & 6 & 3 & 151 & 155 & $\frac{2}{3}$ & $\begin{array}{l}-2 \\
-2\end{array}$ & $\frac{6}{6}$ & $\frac{4}{5}$ & 163 & 165 & \\
\hline-7 & 6 & 5 & 122 & 122 & 2 & -2 & 6 & 6 & $\begin{array}{l}116 \\
1.88\end{array}$ & $\begin{array}{l}111 \\
174\end{array}$ & \\
\hline-7 & 6 & 6 & 249 & 260 & 3 & -2 & 6 & 7 & 145 & 141 & \\
\hline-7 & 6 & 7 & 59 & 60 & 4 & -2 & 6 & 8 & 74 & 78 & \\
\hline-7 & 6 & 9 & 53 & 50 & 5. & -2 & 6 & 9 & 116 & 112 & \\
\hline-7 & 6 & 10 & 106 & 1.01 & 3 & -1 & 6 & 0 & 350 & 355 & \\
\hline-7 & 6 & 11 & 152 & 149 & 3 & -1 & 6 & 1 & 75 & 78 & \\
\hline-6 & 6 & 0 & 263 & 283 & 3 & -1 & 6 & 2 & 79 & 81 & \\
\hline-6 & 6 & 1 & 61 & 54 & 4 & -1 & 6 & 3 & 69 & 61 & \\
\hline-6 & 6 & 4 & 177 & 177 & 3 & -1 & 6 & 4 & 63 & 6.3 & \\
\hline-6 & 6 & 5 & 51 & 46 & 4 & -1 & 6 & 5 & 169 & 166 & \\
\hline-6 & 6 & 7 & 99 & 95 & 3 & -1 & 6 & 6 & 152 & 147 & \\
\hline-5 & 6 & 8 & 280 & 281 & 3 & -1 & 6 & 7 & 60 & 55 & \\
\hline-6 & 6 & 9 & 121 & 123 & 3 & -1 & 6 & 8 & 278 & 270 & \\
\hline-5 & 6 & 0 & 53 & 53 & 4 & -1 & 6 & 9 & 65 & 71 & \\
\hline-5 & 6 & 1 & 86 & 89 & 3 & -1 & 6 & 10 & 68 & 73 & \\
\hline-5 & 6 & 2 & 313 & 329 & 3 & -1 & 6 & 11 & 80 & 80 & \\
\hline-5 & 6 & 3 & 84 & 81 & 3 & 0 & 6 & 0 & 237 & 243 & \\
\hline-5 & 6 & 4 & 142 & 145 & 2 & 0 & 6 & 1 & 240 & 243 & \\
\hline-5 & 6 & 5 & 67 & 70 & 3 & 0 & 6 & 2 & 308 & 305 & \\
\hline-5 & 6 & 6 & 156 & 154 & 2 & 0 & 6 & 3 & 114 & 108 & \\
\hline-5 & 6 & 7 & 49 & 47 & 5 & 0 & 6 & 4 & 45 & 42 & \\
\hline-5 & 6 & 10 & 156 & 154 & 3 & 0 & 6 & 5 & 102 & 93 & \\
\hline-5 & 6 & 11 & 83 & 88 & 4 & 0 & 6 & 6 & 189 & 184 & \\
\hline-4 & 6 & 0 & 297 & 329 & 3 & 0 & 6 & 7 & 116 & 119 & \\
\hline-4 & 6 & 1 & 38 & 45 & 5 & 0 & 6 & 9 & 105 & 1.02 & \\
\hline-4 & 6 & 2 & 87 & 95 & 3 & 0 & 6 & 10 & 176 & 1.72 & \\
\hline-4 & 6 & 3 & 56 & 59 & 4 & 1 & 6 & 0 & 347 & 355 & \\
\hline-4 & 6 & 4 & 153 & 159 & 2 & 1 & 6 & 1 & 92 & 94 & \\
\hline-4 & 6 & 5 & 132 & 133 & 2 & 1 & 6 & 2 & 112 & 108 & \\
\hline-4 & 6 & 6 & 153 & 153 & 2 & 1 & 6 & 3 & 163 & 161 & \\
\hline-4 & 6 & 7 & 96 & 96 & 3 & 1 & 6 & 4 & 270 & 264 & \\
\hline-4 & 6 & 8 & 144 & 140 & 2 & 1 & 6 & 5 & 214 & 214 & \\
\hline-4 & 6 & 9 & 155 & 146 & 2 & 1 & 6 & 7 & 63 & 52 & \\
\hline
\end{tabular}


Values of $10 *$ Fobs and $10 \times \mathrm{Fcal}$ c

Page 19

\begin{tabular}{|c|c|c|c|c|c|c|c|c|c|c|}
\hline $\mathrm{H} \quad \mathrm{K}$ & K. I & Fobs & Fcalc & SigF & H & K & $\begin{array}{l}L_{1} \\
-\end{array}$ & Fobs & Fcalc & $\frac{\operatorname{SigF}}{}$ \\
\hline 1 & 8 & 172 & 174 & 2 & 10 & 6 & 2 & 148 & 142 & 3 \\
\hline 6 & 0 & 79 & 75 & 3 & 10 & 6 & 3 & 85 & 78 & 4 \\
\hline 2 & 1 & 242 & 248 & 3 & 11. & 6 & 0 & 176 & 170 & 3 \\
\hline 2 & 2 & 299 & 293 & 3 & 11 & 6 & 1 & 92 & 92 & 4 \\
\hline 2 & 3 & 137 & 134 & 2 & -10 & 7 & 0 & 48 & 42 & 6 \\
\hline 2 & 4 & 189 & 193 & 3 & -10 & 7 & 1 & 74 & 75 & 4 \\
\hline 2 & 6 & 126 & 119 & 2 & -10 & 7 & 2 & 117 & 115 & 3 \\
\hline 2 & 7 & 84 & 89 & 3 & -10 & 7 & 3 & 169 & 160 & 3 \\
\hline 2 & 510 & 87 & 86 & 4 & -10 & 7 & 5 & 68 & 51 & 4 \\
\hline 3 & 0 & 161 & 168 & 2 & -10 & 7 & 6 & 83 & 73 & 4 \\
\hline 3 & 2 & 103 & 105 & 2 & -9 & 7 & 0 & 40 & 42 & 7 \\
\hline 3 & 3 & 109 & 105 & 2 & -9 & 7 & 1 & 70 & 74 & 4 \\
\hline 3 & 4 & 104 & 106 & 2 & -9 & 7 & 2 & 94 & 96 & 3 \\
\hline 3 & 5 & 199 & 198 & 3 & -9 & 7 & 3 & 86 & 83 & 3 \\
\hline 3 & 6 & 232 & 229 & 3 & -9 & 7 & 4 & 188 & 180 & 3 \\
\hline 3 & 7 & 136 & 135 & 3 & -9 & 7 & 5 & 103 & 95 & 3 \\
\hline 3 & 9 & 102 & 97 & 3 & -9 & 7 & 6 & 64 & 57 & 4 \\
\hline 4 & 0 & 311 & 329 & 3 & -9 & 7 & 7 & 113 & 108 & 3 \\
\hline 4 & 1 & 130 & 139 & 2 & -9 & 7 & 8 & 128 & 126 & 3 \\
\hline 4 & 2 & 37 & 39 & 6 & -8 & 7 & 0 & 88 & 90 & 3 \\
\hline 4 & 3 & 149 & 143 & 2 & -8 & 7 & 1 & 80 & 86 & 3 \\
\hline 4 & 4 & 67 & 67 & 4 & -8 & 7 & 2 & 113 & 117 & 3 \\
\hline 4 & 5 & 98 & 105 & 3 & -8 & 7 & 3 & 124 & 128 & 3 \\
\hline 4 & 6 & 75 & 80 & 4 & -8 & 7 & 6 & 57 & 60 & 5 \\
\hline 6 & 8 & 139 & 135 & 3 & -8 & 7 & 7 & 52 & 48 & 5 \\
\hline 6 & 0 & 50 & 53 & 5 & -7 & 7 & 0 & 48 & 46 & 5 \\
\hline 6 & 2 & 237 & 230 & 3 & -7 & 7 & 1 & 91 & 98 & 3 \\
\hline 5 & 3 & 74 & 76 & 3 & -7 & 7 & 2 & 98 & 100 & 3 \\
\hline 6 & 4 & 46 & 45 & 5 & -7 & 7 & 3 & 89 & 89 & 3 \\
\hline 6 & 5 & 85 & 82 & 3 & -7 & 7 & 4 & 87 & 89 & 3 \\
\hline 6 & 7 & 157 & 152 & 2 & -7 & 7 & 5 & 135 & 129 & 2 \\
\hline 6 & 0 & 282 & 283 & 3 & -7 & 7 & 6 & 11.3 & 115 & 3 \\
\hline 6 & 4 & 152 & 147 & 2 & -7 & 7 & 7 & 66 & 63 & 4 \\
\hline 6 & 5 & 80 & 83 & 4 & -7 & 7 & 8 & 61 & 66 & 5 \\
\hline 6 & 6 & 86 & 82 & 4 & -7 & 7 & 9 & 89 & 91 & 4 \\
\hline 6 & 0 & 114 & 116 & 3 & -6 & 7 & 0 & 106 & 112 & 3 \\
\hline 6 & 2 & 192 & 186 & 3 & -6 & 7 & 1 & 135 & 139 & 2 \\
\hline 6 & 5 & 51 & 34 & 5 & -6 & 7 & 3 & 100 & 1.02 & 3 \\
\hline 6 & 6 & 56 & 58 & 5 & -6 & 7 & 4 & 218 & 217 & 3 \\
\hline 6 & 0 & 52 & 52 & 5 & -6 & 7 & 5 & 65 & 61 & 4 \\
\hline 6 & 1 & 124 & 128 & 3 & -6 & 7 & 7 & 88 & 85 & 3 \\
\hline 6 & 2 & 72 & 65 & 4 & -6 & 7 & 8 & 102 & 97 & 3 \\
\hline 6 & 3 & 73 & 75 & 4 & -5 & 7 & 9 & 54 & 56 & 5 \\
\hline 6 & 4 & 123 & 119 & 3 & -5 & 7 & 5 & 87 & 78 & 3 \\
\hline 6 & 5 & 51 & 48 & 6 & -5 & 7. & 6 & 183 & 177 & 3 \\
\hline 6 & 0 & 152 & 144 & 2 & -5 & 7 & 7 & 44 & 36 & 6 \\
\hline 6 & 1 & 59 & 63 & 5 & -5 & 7 & 8 & 66 & 65 & 4 \\
\hline 6 & 2 & 125 & 122 & 3 & -4 & 7 & 0 & 142 & 159 & 2 \\
\hline 6 & 4 & 64 & 57 & 5 & -4 & 7 & 1 & 151 & 163 & 2 \\
\hline 6 & 1 & $170^{\cdots \cdots}$ & 173 & 3 & -4 & 7 & 2 & 119 & 121 & \\
\hline
\end{tabular}


Values of $10 *$ Fobs and $10 * \mathrm{Fcalc}$

Page 20

\begin{tabular}{|c|c|c|c|c|c|c|c|c|c|c|c|}
\hline H & K & $\underline{L}$ & Fobs & Fcalc & SigF & H & $\mathrm{K}$ & L & Fobs & $F c a l c$ & SigF \\
\hline-4 & 7 & 6 & 160 & 169 & 3 & 3 & 7 & 3 & 105 & 108 & 3 \\
\hline-4 & 7 & 7 & 59 & 54 & 4 & 3 & 7 & 5 & 123 & 120 & 3 \\
\hline-4 & 7 & 8 & 61 & 63 & 5 & 3 & 7 & 7 & 116 & 122 & 3 \\
\hline-4 & 7 & 9 & 101 & 96 & 3 & 4 & 7 & 0 & 158 & 159 & 2 \\
\hline-3 & 7 & 0 & 200 & 216 & 3 & 4 & 7 & 2 & 48 & 47 & 5 \\
\hline-3 & 7 & 1 & 127 & 132 & 2 & 4 & 7 & 3 & 111 & 106 & 3 \\
\hline-3 & 7 & 2 & 68 & 69 & 3 & 4 & 7 & 4 & 69 & 64 & 4 \\
\hline-3 & 7 & 3 & 195 & 194 & 3 & 5 & 7 & 0 & 56 & 44 & 5 \\
\hline-3 & 7 & 4 & 40 & 39 & 5 & 5 & 7 & 1 & .54 & 50 & 5 \\
\hline-3 & 7 & 5 & 126 & 123 & 2 & 5 & 7 & 2 & 215 & 215 & 3 \\
\hline-3 & 7 & 6 & 106 & 95 & 3 & 5 & 7 & 3 & 93 & 90 & 3 \\
\hline-3 & 7 & 8 & 141 & 140 & 3 & 5 & 7 & 5 & 136 & 127 & 3 \\
\hline-2 & 7 & 0 & 43 & 38 & 4 & 5 & 7 & 6 & 91 . & 85 & 4 \\
\hline-2 & 7 & 1 & 113 & 110 & 2 & 6 & 7 & 0 & 113 & 112 & 3 \\
\hline-2 & 7 & 2 & 275 & 273 & 3 & 6 & 7 & 2 & 89 & 89 & 3 \\
\hline-2 & 7 & 3 & 221 & 216 & 3 & 6 & 7 & 3 & 81 & 74 & 4 \\
\hline-2 & 7 & 4 & 133 & 134 & 2 & 6 & 7 & 4 & 149 & 145 & 2 \\
\hline-2 & 7 & 5 & 211 & 200 & 3 & 6 & 7 & 5 & 68 & 59 & 5 \\
\hline-2 & 7 & 6 & 116 & 110 & 3 & 7 & 7 & 0 & 48 & 46 & 6 \\
\hline-2 & 7 & 7 & 106 & 109 & 3 & 7 & 7 & 1 & 111 & 105 & 3 \\
\hline-2 & 7 & 8 & 118 & 115 & 3 & 7 & 7 & 2 & 96 & 93 & 3 \\
\hline-1 & 7 & 1 & 56 & 54 & 3 & 7 & 7 & 4 & 44 & 49 & 7 \\
\hline-1 & 7 & 2 & 192 & 190 & 3 & 8 & 7 & 0 & 90 & 90 & 3 \\
\hline-1 & 7 & 3 & 200 & 189 & 3 & 8 & 7 & 2 & 80 & 82 & 4 \\
\hline-1 & 7 & 4 & 167 & 161 & 3 & 8 & 7 & 3 & 80 & 72 & 4 \\
\hline-1 & 7 & 5 & 97 & 95 & 3 & 9 & 7 & 0 & 46 & 42 & 6 \\
\hline-1 & 7 & 7 & 120 & 119 & 3 & 9 & 7 & 2 & 67 & 67 & 5 \\
\hline-1 & 7 & 9 & 43 & 28 & 6 & 10 & 7 & 0 & 44 & 42 & 7 \\
\hline 0 & 7 & 3. & 43 & 37 & 4 & -7 & 8 & 1 & 113 & 115 & 3 \\
\hline 0 & 7 & 3 & 84 & 88 & 3 & -7 & 8 & 2 & 85 & 89 & 4 \\
\hline 0 & 7 & 4 & 118 & 114 & 2 & -7 & 8 & 3 & 60 & 56 & 5 \\
\hline 0 & 7 & 5 & 87 & 91 & 3 & -7 & 8 & 4 & 175 & 179 & 3 \\
\hline 0 & 7 & 6 & 67 & 56 & 4 & -6 & 8 & 0 & 42 & 49 & 6 \\
\hline 0 & 7 & 7 & 79 & 80 & 4 & -6 & 8 & 3 & 80 & 71 & 4 \\
\hline 0 & 6 & 7 & 116 & 119 & 2 & -6 & 8 & 5 & 85 & 81 & 4 \\
\hline 0 & 7 & 8 & 54 & 47 & 5 & -5 & 8 & 0 & 163 & 176 & 2 \\
\hline 0 & 7 & 9 & 52 & 47 & 6 & -5 & 8 & 1 & 42 & 35 & 6 \\
\hline 1 & 7 & 1 & 254 & 253 & 3 & -5 & 8 & 2 & 91 & 89 & 3 \\
\hline 1 & 7 & 2 & 87 & 83 & 3 & -5 & 8 & 3 & 64 & 64 & 4 \\
\hline 1 & 7 & 3 & 108 & 115 & 3 & -5 & 8 & 4 & 81 & 83 & 4 \\
\hline 1 & 7 & 4 & 140 & 134 & 2 & -4 & 8 & 0 & 66 & 71 & 4 \\
\hline 1 & 7 & 6 & 58 & 55 & 4 & -4 & 8 & 1 & 64 & 53 & 4 \\
\hline 2 & 7 & 1 & 39 & 40 & 5 & -4 & 8 & 2 & 112 & 113 & 3 \\
\hline 2 & 7 & 3 & 206 & 202 & 3 & -4 & 8 & 3 & 72 & 61 & 4 \\
\hline 2 & 7 & 4 & 88 & 76 & 3 & -4 & 8 & 5 & 109 & 106 & 3 \\
\hline 2 & 7 & 5 & 187 & 179 & 3 & -3 & 8 & 1 & 47 & 47 & 5 \\
\hline 2 & 7 & 6 & 127 & 118 & 3 & -3 & 8 & 2 & 72 & 62 & 3 \\
\hline 2 & 7 & 7 & 171 & 167 & 3 & -3 & 8 & 3 & 59 & 58 & 4 \\
\hline 2 & 7 & 8 & 82 & 82 & 4 & -3 & 8 & 6 & 100 & 94 & 3 \\
\hline 3 & 7 & 0 & 214 & 216 & 3 & -2 & 8 & 0 & 170 & 175 & 3 \\
\hline
\end{tabular}


Values of $10 \times$ Fobs and $10 *$ Falc

\begin{tabular}{|c|c|c|c|c|c|c|c|c|c|c|c|}
\hline H & K & $\stackrel{L}{-}$ & Fobs & Fod 10 & $\operatorname{SigF}$ & $\mathrm{H}$ & K & $\mathrm{I}$ & Fobs & Fod 10 & SigF \\
\hline
\end{tabular}

$\begin{array}{rrrrrr}-2 & 8 & 1 & 201 & 197 & 3 \\ -2 & 8 & 2 & 80 & 77 & 3 \\ -2 & 8 & 3 & 56 & 53 & 4 \\ -2 & 8 & 6 & 72 & 59 & 4 \\ -1 & 8 & 2 & 77 & 63 & 3 \\ -1 & 8 & 3 & 184 & 181 & 3 \\ -1 & 8 & 4 & 99 & 91 & 3 \\ -1 & 8 & 5 & 109 & 106 & 3 \\ -1 & 8 & 6 & 43 & 43 & 6 \\ 0 & 8 & 1 & 97 & 90 & 3 \\ 0 & 8 & 3 & 41 & 30 & 6 \\ 0 & 8 & 5 & 97 & 85 & 3 \\ 1 & 8 & 1 & 116 & 112 & 3 \\ 1 & 8 & 2 & 79 & 75 & 3 \\ 1 & 8 & 4 & 52 & 51 & 5 \\ 1 & 8 & 5 & 102 & 96 & 3 \\ 1 & 8 & 6 & 51 & 42 & 6 \\ 2 & 8 & 0 & 179 & 175 & 3 \\ 2 & 8 & 2 & 108 & 94 & 3 \\ 2 & 8 & 3 & 96 & 93 & 3 \\ 2 & 8 & 4 & 102 & 100 & 3 \\ 3 & 8 & 1 & 168 & 164 & 3 \\ 3 & 8 & 2 & 164 & 162 & 3 \\ 3 & 8 & 3 & 49 & 45 & 6 \\ 3 & 8 & 4 & 82 & 79 & 4 \\ 4 & 8 & 0 & 69 & 71 & 4 \\ 4 & 8 & 2 & 42 & 35 & 6 \\ 4 & 8 & 3 & 136 & 123 & 3 \\ 5 & 8 & 0 & 172 & 176 & 3 \\ 5 & 8 & 1 & 70 & 72 & 4 \\ 6 & 8 & 1 & 102 & 92 & 3 \\ & & & & & \end{array}$

